\title{
KINETIC THEORY ANALYSIS OF RAREFIED GAS FLOW THROUGH FINITE LENGTH SLOTS
}

by

Pattabiraman Raghuraman

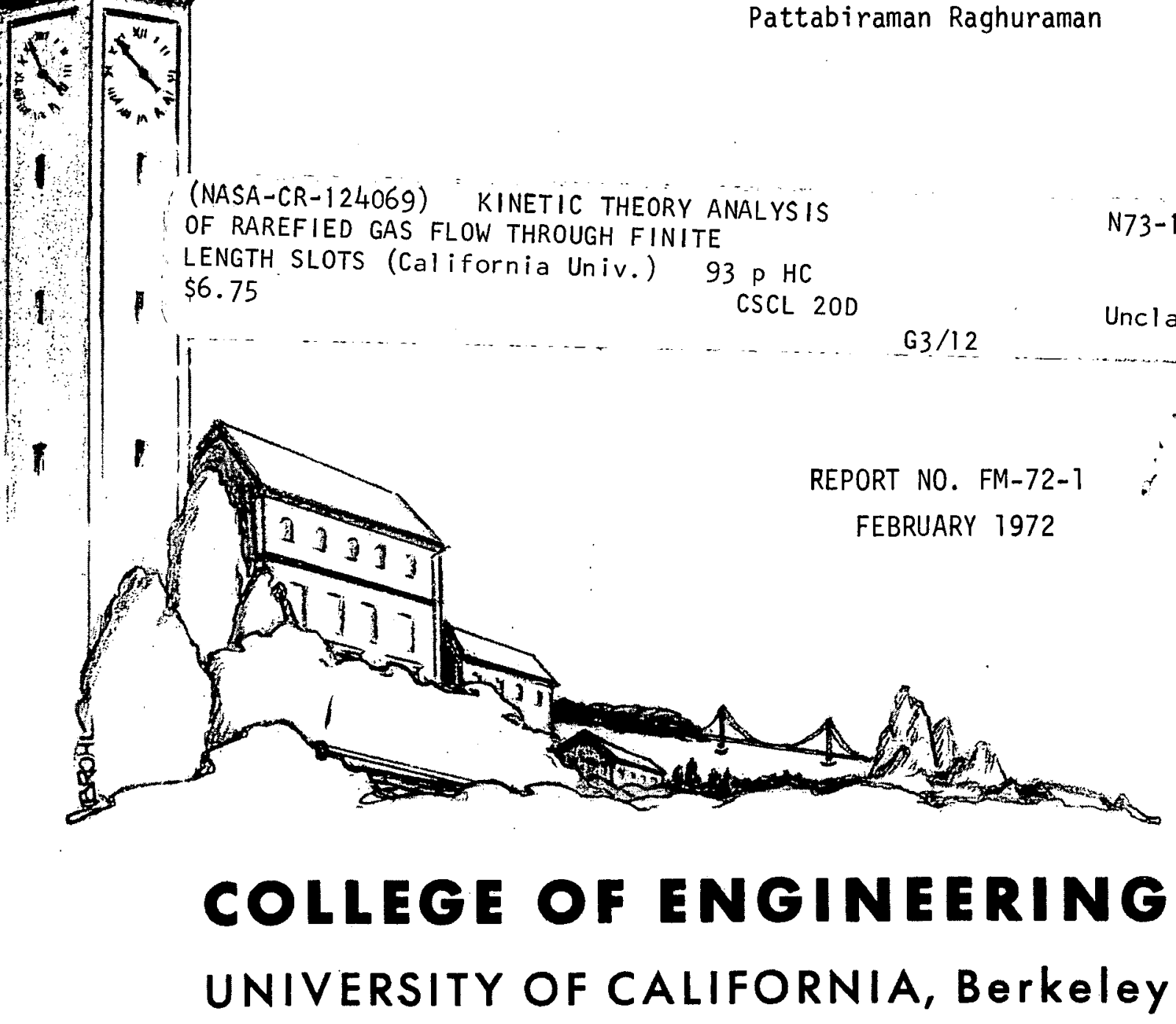


REPORT NO. FM-72-1

FEBRUARY 1972

KINETIC THEORY ANALYSIS OF RAREFIED GAS FLOW THROUGH FINITE LENGTH SLOTS

by

Pattabiraman Raghuraman 
The early stages of this research were supported by the National Aeronautics and Space Administration under Contract NAS-8-21432, "Lunar Surface Engineering Properties Experiment Definition," for the Marsha11 Space Flight Center, Huntsville, Alabama.

Support for this work was also received from the Office of Naval Research and the National. Science Foundation.

Publication was accomplished under NSF Grant GK-11651, Amend. I. 
An analytic study is made of the flow of a rarefied monatomic gas through a two dimensional slot. The parameters of the problem are the ratios of downstream to upstream pressures; $\alpha$, the Knudsen number at the high pressure end (based on slot half width) $\mathrm{Kn}_{0}$, and the length to slot half width ratio, $\ell$. First, a moment method of solution is used by assuming a discontinuous distribution function consisting of four Maxwellians split equally in angular space. Numerical solutions are obtained for the resulting equations. The characteristics of the transition regime are portrayed very we11; however the solutions in the free molecule limit are systematically lower than the results obtained in that limit by more accurate numerical methods.

Finally, the discrete velocity ordinate method of solution is used. The continuous velocity space is represented by 16 ordinates. Numerical calculations are used to obtain the characteristics of the transition regime. These characteristics are very well represented by this method. Further, the free molecule and the slip regime results appear to be highly accurate and serve to bolster further confidence in the accuracy of the transition regime results.

For the range of parameters considered, the mass flux through the slot, $\dot{m}$, is given by,

$$
\begin{aligned}
& m / \rho_{0} a\left(2 R T_{0}\right)^{1 / 2}=\frac{1}{\ell}\left\{\frac{0.1331}{K n_{0}}(1+\alpha) T_{1}+2\left(T_{1}-\frac{K n_{0}}{8} \log s_{1}\right)\right. \\
& +\frac{K n_{0}}{8 \sqrt{\pi}} \log s_{1} \log \left(\frac{\ell+\sqrt{\ell^{2}+4}}{2}\right) \text {, }
\end{aligned}
$$


where,

$$
\begin{aligned}
& T_{1}=\frac{(1-\alpha) \ell}{\ell+4.55-2.85 \exp \left\{0.2 T\left(K n_{0}-5\right)\right\}} \\
& s_{1}=\frac{2 K n_{0}+8(1+\alpha+T)}{2 K n_{0}+8(1+\alpha-T)}
\end{aligned}
$$

$R$ is the gas constant, ' $a$ ' the slot half width, $\rho_{0}$ and $T_{0}$ the upstream density and temperature, respectively. The accuracy of this formula, compared to the numerical results obtained, is $5 \%$. It is valid for $\ell=1,2,4,8$ and $12, \alpha=0.1,0.5$ and 0.8 and $K n_{0}=\infty, 5,1$ and 0.5 
ABSTRACT

LIST OF FIGURES v v

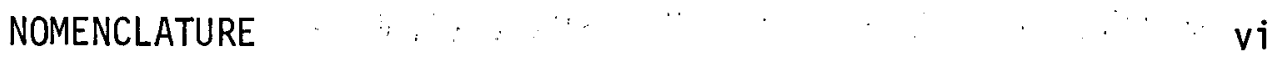

1.0 INTRODUCTION , $\cdots, \cdots \cdots$

1.1 Review of Previous Theoretical Work 2

1.2 Review of Available Theoretical Tools 3

1.3 The Present Investigation 5

2.0 STATEMENT OF THE PROBLEM AND ASSUMPTIONS 8

2.1 Governing Equations and Boundary Conditions 9

$\begin{array}{ll}3.0 & \text { MOMENT METHOD OF SOLUTION }\end{array}$

3.1 Formulation 16

3.2 Free Molecule Solution 20

3.3 Transition Flow Calculations 21

4.0 DISCRETE VELOCITY ORDINATE METHOD OF SOLUTION 26

4.1 Formulation 26

4.2 Free Molecule Limit Solution 34

4.3 Transition Flow Calculations 38

5.0 DISCUSSION OF SOLUTIONS

5.1 Free Molecule Results 43

5.2 Transition Flow Results 44

5.3 Improvements and Suggestions for Future Work 48

$\begin{array}{ll}6.0 \text { CONCLUSIONS } & 50\end{array}$

REFERENCES $\quad 52$

FIGURES 
APPENDIX A. EXISTENCE AND UNIQUENESS OF SOLUTION OF

MOMENT EQUATIONS

APPENDIX B. FREE MOLECULE FLOW RESULTS FOR THE MOMENT

METHOD

75

APPENDIX C. ITERATION SCHEME FOR INITIAL VALUES

77

APPENDIX D. GAUSSIAN QUADRATURE FORMULAS FOR

$\int_{0}^{\infty} d c c e^{-c^{2}} h_{1}(c)$ 


\section{LIST OF FIGURES}

Fig. la Slot Geometry

Fig. Ib Slot Geometry

Fig. 2 Grid Points for the Moment Method

Fig. 3 Grid Points for the Discrete Ordinate Method

Fig. 4 Comparison of Slot Mass Flux vs. $\ell$ by the Various Solutions for $\mathrm{Kn}_{\mathrm{o}}=\infty$

Fig. 5 Free Molecule Wall Flux vs. $x, \ell=4, \alpha=0$

Fig. 6 Free Molecule Wa11 No Flux vs. $x, \ell=8, \alpha=0$

Fig. 7 Free Molecule Wall Flux vs. $x, i=4, \alpha=0$

Fig. 8 Free Molecule Wall : No Flux vs. $x, \ell=8, \alpha=0$

Fig. 9 Wall No Flux vs. $x_{2} \cdot \ell=1, \alpha=0.1 \& \mathrm{Kn}_{0}=0.5$

Fig. 10 Wall No Flux vs. $x, \ell=4, \alpha=0.1, \mathrm{Kn}_{0}=1.0 \& 5.0$

Fig. 11 Wall No Flux vs. $\dot{x}, \ell=4, \alpha=0.7, \quad k n_{0}=1.0 \& 5.0$

Fig. 12 Wall No Flux vs. $x, l=12, \alpha=0.1, k n_{0}=5.0,1.0$.

Fig. 13 Interpolation of Mass Flux

Fig. 14 ou vs. $y, . \ell=12, \alpha=0.1 \& \mathrm{Kn}_{0}=5.0$

Fig. 15 ou vs. $y, \quad \ell=12, \alpha=0.1 \& K n_{0}:=1.0$ 


\section{NOMENCLATURE}

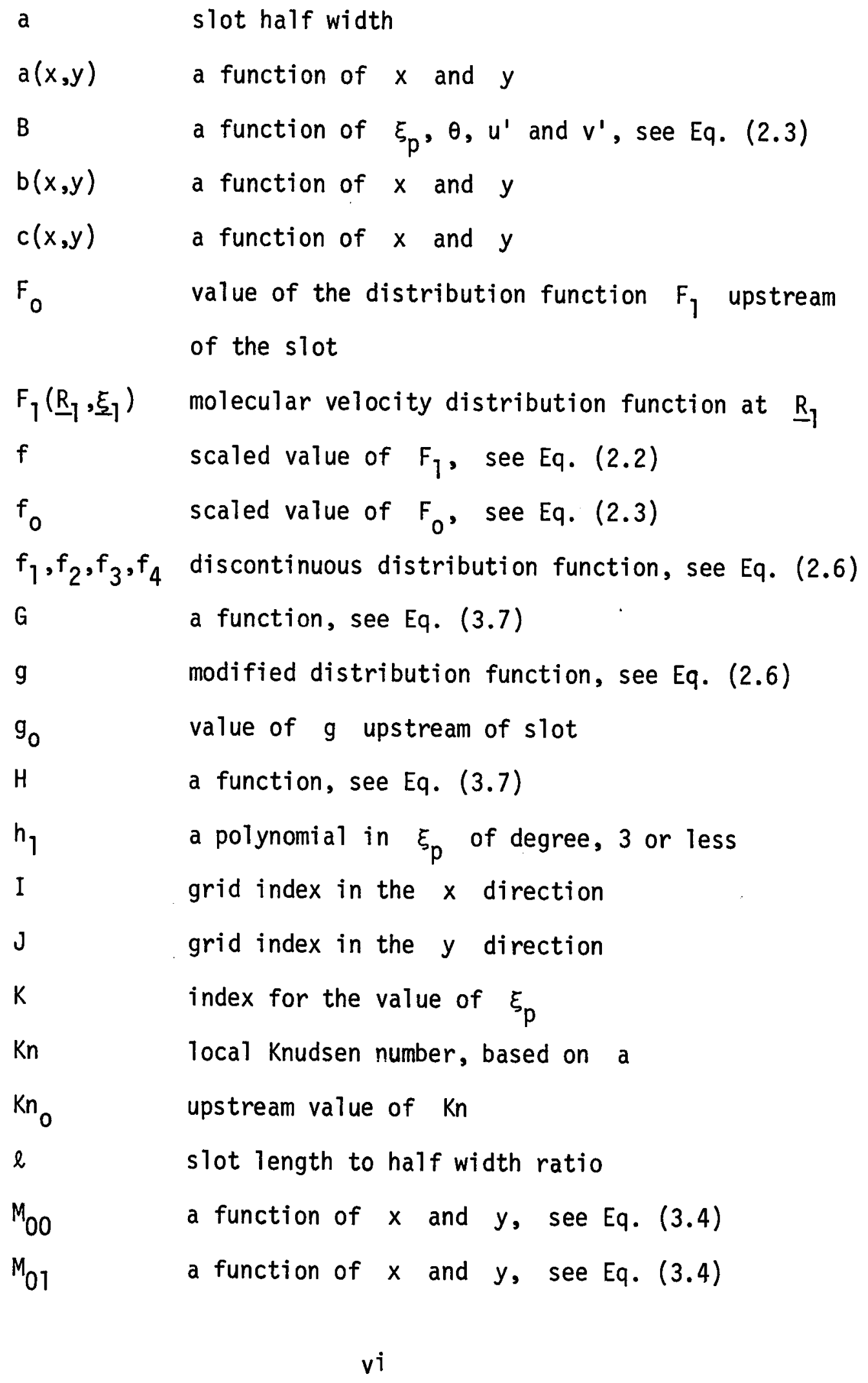




\begin{tabular}{|c|c|}
\hline$M_{10}$ & a function of $x$ and $y$, see Eq. (3.4) \\
\hline$M_{11}$ & a function of $x$ and $y$, see Eq. (3.4) \\
\hline $\mathrm{m}$ & mass of a gas molecule \\
\hline$\dot{\mathrm{m}}$ & mass flux through the slot \\
\hline N & number of grid points along the slot length \\
\hline$n$ & index for the value of $\theta$ \\
\hline NN & number of grid points at slot entrance \\
\hline$P^{\prime}$ & a function of $x$ and $y$, see Eq. (3.14) \\
\hline$P_{i j}$ & $\begin{array}{l}\text { flux of } i \text { component momentum of gas in the } j \text { th } \\
\text { direction }(i, j=x, y, z)\end{array}$ \\
\hline$p_{0}$ & pressure of gas upstream of slot \\
\hline Q & value of $\dot{m}$ scaled by $\rho_{0} a\left(2 R T_{0}\right)^{1 / 2}$ \\
\hline$Q\left(\xi_{p}, \theta\right)$ & a separable function of $\xi_{p}$ and $\theta$ \\
\hline$Q^{\prime}$ & a function of $x$ and $y$, see Eq. (3.14) \\
\hline$Q_{1}\left(\xi_{p}\right)$ & a function of $\xi_{p}$ \\
\hline$Q_{2}(\theta)$ & a function of $\theta$ \\
\hline R & gas constant \\
\hline $\mathrm{R}_{1}$ & physical space position vector \\
\hline$R^{\prime}$ & a function of $x$ and $y$, see Eq. (3.14) \\
\hline $\mathrm{S}_{1}$ & a function of $\ell, \alpha$ and $k n_{0}$ \\
\hline$S^{\prime}$ & a function of $x$ and $y$, see Eq. (3.14) \\
\hline$T\left(\underline{R}_{1}\right)$ & temperature of gas \\
\hline $\mathrm{T}_{0}$ & temperature of gas upstream and downstream of slot \\
\hline $\mathrm{T}_{1}$ & a function of $\ell, \alpha$ and $k n_{0}$ \\
\hline $\begin{array}{l}u \\
u^{\prime}\end{array}$ & $\begin{array}{l}\text { macroscopic velocity of gas along } x 1 \text { direction } \\
\text { value of } u \text { scaled by }\left(2 \mathrm{RT}_{0}\right)^{1 / 2}\end{array}$ \\
\hline
\end{tabular}


macroscopic velocity of gas along y1 direction value of $v$ scaled by $\left(2 R_{0}\right)^{1 / 2}$ macroscopic velocity of gas along $z 1$ direction Gaussian weights, defined in Appendix $D$ value of $x l$ scaled by a distance along slot length from slot entrance value of yi scaled by a distance from slot centerline value of zl scaled by a distance normal to $x]$ and $y l$ directions ratio of downstream to upstream pressure increment in the initial value guesses direction of velocity vector of a gas molecule in the $(x, y)$ plane collision frequency speed of a molecule Gaussian root values of $\xi_{p}$, defined in Appendix $D$ speed of a gas molecule in $(x, y)$ plane, scaled by $\left.(2 \mathrm{RT})_{0}\right)^{1 / 2}$

velocity of molecules along $x 1$ direction velocity of molecules along yl direction value of $\xi_{z 1}$ scaled by $\left(2 R_{0}\right)^{1 / 2}$ velocity of molecule along $z l$ direction velocity vector of a molecule speed of a gas molecule 


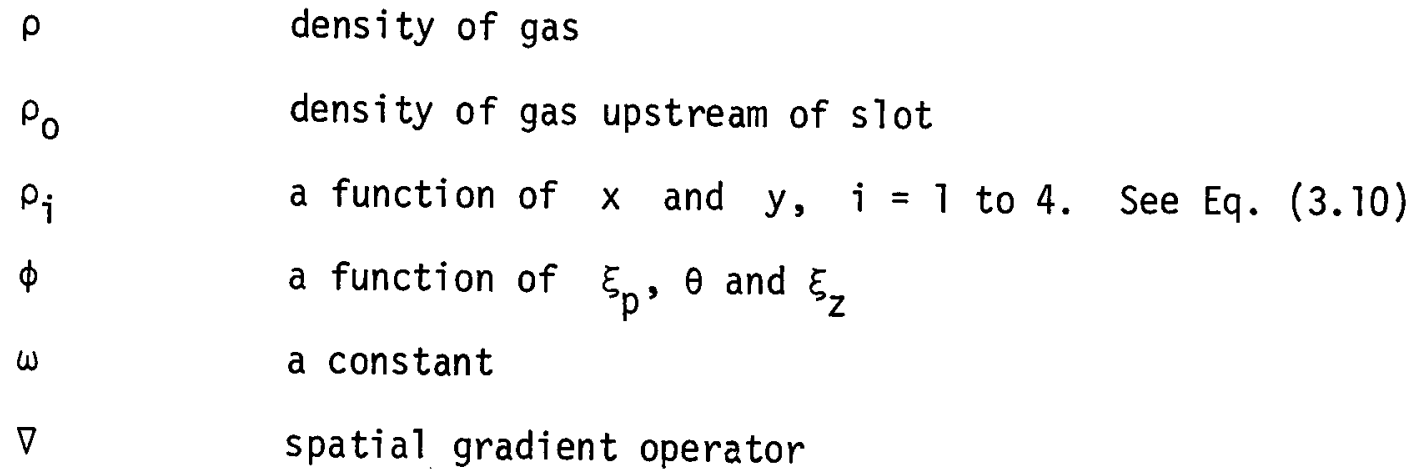




\subsection{INTRODUCTION}

From a fluid mechanics viewpoint one of the most attractive features of kinetic thoery (with the Boltzmann equation as the fundamental equation) is its ability to predict the macroscopic properties of a dilute gas in terms of its microscopic properties under highly nonequilibrium situations. If 'appropriate' boundary conditions are carefully specified, it can be used for both internal and external flows. The investigation reported in this thesis is a kinetic theory study of the flow of a dilute monatomic gas down a finite length (length of slot along direction of flow) slot for arbitrary ratios of pressures applied at the ends of the slot.

The flow first came into focus in the design of pressure probes to measure the permeability of lunar surface soil. The design features involved the pumping of a gas from a high pressure into the soil and carried the assumption that the pressure in the flow dropped off to zero far from the probe. If the porous medium were visualized as an assembly of cracks and holes, our solution could be relevantly applied to the former geometry. It is also anticipated that such a flow situation could occur on the Earth's soil in places having high pressure gas pockets which are connected to the atmosphere through tiny cracks.

As the mean free path at the high pressure end of the slot is varied, it is expected that, if the length of the slot is sufficiently long, the flow will pass through all stages (or some stages) of rarefaction (characterized by the Knudsen number which is the ratio of the mean free path of the gas to the slot half width ${ }^{*}$ ). ${ }^{\star} \mathrm{Half}$ the distance between the slot walls. 
The above system represents a fundamental flow problem. Rather loosely, it can be compared to the problem of the free jet. Whereas no solid boundaries are involved there, the slot problem, representing an equally nonequilibrium system, has solid boundaries playing a decisive role in the flow. The flow is a highly nonlinear one, and the number of such highly nonlinear problems that have been considered using kinetic theory has been relatively few. In view of these considerations, a thorough kinetic theory investigation of the flow is considered.

\subsection{Review of Previous Theoretical Work}

In spite of the fundamental nature of the geometry and the flow, a solution of sufficient rigor and generality does not seem available.

For free molecule flow through slots of finite length, Reynolds and Richey (1967) perform accurate numerical calculations for various flow properties. Perhaps the most striking result here is that the number flux from the wall varies essentially linearly along the length of the slot. A variational solution is also available due to Pao and Willis (1962). For flow in the slip regime, the only prevailing result is the Poiseuille formula with slip boundary conditions. This result, however, is valid only for long slots and no corresponding result is available for slots of arbitrary length.

For slots of infinite length, various calculations in the transition regime have been performed. Cercignani and Daneri (1963) numerically solved the BGK equation and demonstrated the 
existence of a 'Knudsen minimum' for the linearized flow (with small pressure gradients along the slot axis). The same problem was also treated by Liu (1968), using the Moment method with a choice of the form of the distribution function. It consisted of a discontinuous distribution made up of four Maxwellians split equally in angular space. Finally, an ad hoc interpolation formula, propounded by Hilby and Pahl (1952), is available. It involves the interpolation of free molecule and slip flow results to transition flow. No comparable results exist for slots of finite length.

\subsection{Review of Available Theoretical Tools}

The Boltzmann equation is valid for arbitrary deviations from equilibrium. However, the complicated mathematical structure of the collision term has limited direct use to asymptotic values of the Knudsen number. In the last few years a series of different methods were used to obtain approximate solutions in the transition regime. Some of the methods that could be applied to our problem are reviewed briefly below.

One approach is to use the Moment method. Here the Maxwell transport equation replaces the Boltzmann equation as the governing equation with the understanding that the exact solution of the Boltzmann equation is one that satisfies the Maxwell equation for all its moments. However, the solution of an infinite set of equations is not feasible. Hence a truncation of the equations is performed to get a closed set of equations. This can be done through realistic physical assumption--see for example Hamel and 
Willis (1966). The alternative approach is to recognize that the distribution function is formally a function of two sets of independent variables, the physical space position vector $\underline{R}_{1}$ and the velocity space position vector $\xi_{\rceil}$. An explicit form of the $\xi_{7}$ dependence is assumed, which is compatible with the boundary conditions. The $\underline{R}_{1}$ dependence of the distribution function is still an unknown. This is emphasized by the presence of a number of unknown functions of $\underline{R}_{1}$ in the form of distribution function assumed. The number of such unknown functions of $\underline{R}_{1}$ required should be enough to form a determinate set of equations made up of the conservation equations and a reasonable number of higher moment equations to represent rarefaction effects. A satisfactory solution is hard to define exactly but should certainly represent correctly the asymptotic cases of high and low knudsen number. This method is advocated by Lees. A review of the method and applications to some flow problems is given in Lees (1965).

A frequent practice is to replace the collision operator in the Boltzmann equation by a relaxation model to give the Bhatnagar, Gross and Krook (BGK) equation (1954). Direct numerical solution of the BGK equation has been successfully attempted--see for example Anderson (1967) and Liepmann, Narasimha and Chahine (1962). For linearized internal flows, direct integration of the BGK equation has been performed by Cercignani and Daneri (1963). For such flows, variational solutions have also been successfully obtained by Cercignani and co-workers--see for example Cercignani and Pagani (1966). 
A powerful approach first used in applications in radiative transfer is the 'discrete ordinate' method. It has been widely applied by Huang and his.co-workers in transition flow, both for one and two dimensional, internal and external flow problems--see for example Huang and Hartley (1969) and the references cited there. In this method, the continuous velocity. space is replaced by a number of discrete points to generate a system of equations for the velocity distribution function at these discrete values of the velocity. From the evaluation of these distribution functions, any physical quantity can be calculated.

Also worthy of attention is the 'Restricted Variational Method' propounded first by Rosen (1954). Here the task of solving the Boltzmann equation is replaced by one of optimizing an integral. A form of the distribution function has to be assumed and succeeding steps closely follow the Rayleigh-Ritz technique. The method has since been successfully used by Ortloff (1968) as well.

A powerful technique for solving the Boltzmann equation is the Monte Carlo method. This has been most effectively. employed by Bird (1969) and his co-workers. However, the development of such a method involves a considerable amount of computer program development and comparatively large running times to obtain accurate results.

\subsection{The Present Investigation}

At the outset a simple discontinuous distribution function, made up of four Maxwellians split equally in angular space, is assumed and the Moment method used. The resulting set of semi- 
linear hyperbolic partial differential equations is integrated using the method of characteristics. In the free molecule limit (where the Knudsen number based on the slot half width $\rightarrow \infty$ ) an analytical solution is obtained. While the gross features of the flow are correctly represented, the free molecule mass flux is systematically lower (for the ranges of the various parameters considered) than that reported by Reynolds and Richey (1967). In fact, for a slot length to half width ratio of 12 , the difference is almost $35 \%$ and it is expected that as this slot length to half width ratio increases, the difference would get larger. Also the method, as evidenced by the results, seems suitable only for slot lengths to half width ratios between 6 and 12. Results in the transition regime, for upstream Knudsen numbers less than, or equal to, 5 and slot lengths to half width ratios between 1 and 12 seem reasonable. Also for low values of the Knudsen number, i.e., approaching the continuum regime, the results agree remarkably well with the Poiseuille flow formula using slip boundary conditions.

Due to the deficiency of the Moment method in the free molecule limit, a discrete velocity method solution of the BGK equation is attempted next. The unknown is a modified distribution function. The temperature is set equal to a constant, with negligible errors expected based on the moment method estimates. The velocity space is represented by 16 discrete velocities and the distribution function is evaluated at these points by numerical calculations. Two distinct methods are used for integrating for 
the normal mass flux at the wall and the macroscopic quantities at any point. The first scheme is a simple trapezoidal rule. The second integration scheme allows for the free molecule discontinuity of the distribution function and accounts for a linear variation along the slot length of the number flux from the walls in the free molecule limit. In the free molecule limit, the former method leads to overestimates of the mass flux for large slot lengths--as much as $35 \%$ for a slot length to half width ratio of 12 . The latter method of integration has errors of less than $0.1 \%$ for a slot length to half width ratio ranging from 1 to 12 . The differences between the two modes of integration decrease as the Knudsen number decreases, being only $5.0 \%$ for length to slot half width ratios of 1 to 12 , pressure ratios ranging from 0.1 to 0.8 , and upstream Knudsen number less than 5. 


\subsection{STATEMENT OF THE PROBLEM AND ASSUMPTIONS}

Consider a slot such as is shown pictorially in Fig. 1a. The dimension of the slot $l a$ in the direction of the flow is called the length of the slot. The dimension of the slot $2 a$ between the two walls is called the slot width. The slot has infinite. depth (the dimension normal to the slot width and length). The coordinate system is oriented as shown in Fig. la with walls of the slot being represented by $y 1= \pm a, 0 \leqslant x 1 \leqslant \ell a$... Figure. $1 b$ shows a section (parallel to the flow) of the slot. The slot separates two reservoirs containing the same monatomic gas at a temperature $T_{0}$. However, on the side $\left.x\right]=0$, the density is $\rho_{0}$, the pressure is $p_{0}\left(=\rho_{0} R T_{0}\right.$, with $R$ the gas constant $)$ and the Knudsen number is $K n_{0}$ (based on the slot half width ' $a$ '). On the side $x]=a l$, the pressure is $\alpha p_{0}$ where $\alpha$ is a nondimensional constant with a value less than 1 .

We make the assumption that the incoming stream on either side of the slot is known. These are taken as the distribution function in the corresponding reservoirs, i.e., Maxwellians. This is an approximation since the distribution functions of the molecules coming into the ends of the slot from either reservoir is going to be altered by molecules coming out of the slot into the reservoirs. There is, however, no easy way of estimating the degree of this effect (and hence the errors involved by our assumption) unless we solve for the whole flow field. This could be a topic for future research.

Further, there is no accumulation or ablation at the slot 
walls. Finally, the molecules are assumed to be remitted from the wall diffusely at temperature $\mathrm{T}_{0}$.

The problem as posed is to determine the flow field in the slot and in particular the mass flux through the slot. Onily half (above the slot axis $y 1=0$ ) of the flow field need be" considered due to the symmetry of the flow about the plane $y l^{\prime}=0$.

\subsection{Governing Equations and Boundary Conditions}

Let us denote the physical position vector by $\underline{R}_{1}=$ $(x], y 1, z])$ and the velocity vector of a molecule by $\underline{\xi}_{j}=$ $\left(\xi_{x 1}, \xi_{y 1}, \xi_{z 1}\right)$, where $\xi_{x l}, \xi_{y 1}$ and $\xi_{z 1}$ are the velocity components along the $x 1, y 1$ and $z 1$ axis, respectively. The molecular velocity distribution function $F_{1}\left(\underline{R}_{1}, \underline{\xi}_{j}\right)$ is defined to be the number of molecules per unit of volume of the $\left(\underline{R}_{7}, \underline{\xi}_{\rceil}\right)$space: The mass density $\rho$, velocity $\underline{U}=(u, v, w)$, temperature $T$ and $P_{i j}$, the flux of ' $i$ ' component momentum in the $' j \cdot$ ' direction, for the monatomic gas medium; are defined by the following moments of $F_{1}\left(\underline{R}_{1}, \underline{\xi}_{j}\right)$ :

$$
\begin{aligned}
& \rho\left(\underline{R}_{1}\right)=m \iiint d^{3} \xi_{1} F_{1} \\
& \rho \underline{U}\left(\underline{R}_{1}\right)=m \iiint d^{3} \xi_{1} \xi_{7} F_{1} \\
& 3 \rho R T\left(\underline{R}_{7}\right)=m \iiint d^{3} \xi_{1}\left(\underline{\xi}_{7}-\underline{U}\right)^{2} F_{1},
\end{aligned}
$$

and

$$
P_{i j}\left(\underline{R}_{1}\right)=m \iiint d^{3} \xi_{1} \xi_{i} \xi_{j} F_{1}, \quad(i, j=x 1, y 1, z 1),
$$

where

$$
d^{3} \xi_{1}=d \xi_{x 1} \cdot d \xi_{y 1} \cdot d \xi_{z 1},
$$


$m$ is the mass of a molecule, $R$ is the gas constant and the integration is over the whole velocity space. The flow being two dimensional, $W=0$ and $F_{1}$ and hence all its moments are functions independent of $z 1$ (the coordinate normal to the $x l$ and $y 1$ axes). In the steady state with no external force the governing equation for $F_{1}$ can be written as

$$
\underline{\xi}_{1} \cdot \underline{\nabla F}_{1}=\left(\frac{\delta F_{1}}{\delta t}\right)_{\text {coll }}
$$

where $\nabla$ is the spatial gradient operator, and $\left(\delta F_{l} / \delta t\right)_{\text {coll is }}$ the contribution from molecular collisions. In general $\left(\delta F_{1} / \delta t\right)_{c o l l}$ is a nonlinear function of $F_{1}$. For a dilute gas the Maxwell Boltzmann collision integral is the appropriate choice for $\left(\delta F_{1} / \delta t\right)_{\text {coll }}$ (for example, see Kennard, 1938). For mathematical simplicity, however, the Boltzmann collision integral is replaced by a relaxation type model--the BGK equation (1954). In this model equation we write

$$
\left(\frac{\delta F_{1}}{\delta t}\right)_{c 011}=\nu\left[F_{0}-F_{1}\right]
$$

where $\nu$ is the collision frequency which is dependent only on $\underline{R}_{1}$ and

$$
F_{0}=\frac{\rho}{m}(2 \pi R T)^{-3 / 2} \exp \left\{-\left(\underline{\xi}_{j}-\underline{U}\right)^{2} / 2 R T_{0}\right\} .
$$

Thus the equation considered is

$$
\xi_{x 1} \frac{\partial F_{1}}{\partial x T}+\xi_{y 1} \frac{\partial F_{1}}{\partial y T}=\nu\left[F_{0}-F_{1}\right]
$$

In view of the assumptions, the boundary conditions are: 
At $\quad x]=0,-a<y 1<a$ and $\cdot \xi_{x 1}>0$,

$$
F_{1}=\frac{\rho_{0}}{m}\left(2 \pi R T_{0}\right)^{-3 / 2} \exp \left(-\xi_{1}{ }^{2} / 2 R T_{0}\right) \text {, where } \xi_{1}^{2}=\underline{\xi}_{1} \cdot \underline{\xi}_{1} ;
$$

At $\quad x 1=\ell \cdot a,-a<y 1<a$ and $\xi_{x 1}>0$,

$$
F_{1}=\frac{\alpha \cdot \rho_{0}}{m}\left(2 \pi R T_{0}\right)^{-3 / 2} \exp \left(-\xi_{1}^{2} / 2 R T_{0}\right) ;
$$

At $y 1=a, \quad 0<x 1<\ell \cdot a$,

$$
v=0 \text {, i.e., } \iiint d^{3} \xi_{1} \xi_{y 1} F_{1}=0
$$

and

$$
F_{1}=\frac{\rho}{m}\left(2 \pi R T_{0}\right)^{-3 / 2} \exp \left(-\xi_{1}{ }^{2} / 2 R T_{0}\right) \text { for } \xi_{y 1}<0 \quad \text {. }
$$

At $y 1=0$, by symmetry about the plane of the slot axis,

$$
F_{1}\left(\xi_{x 1}, \xi_{y 1}, \xi_{z 1} ; x 1,0\right)=F_{1}\left(\xi_{x 1},-\xi_{y 1}, \xi_{z 1} ; x 1,0\right)
$$

The collision frequency $\nu$ is defined as $\nu=\rho R T / \mu$, where the viscosity $\mu$ is assumed to have the temperature dependence $\mu / \mu_{0}=\left(T / T_{0}\right)^{\omega}$, and $\omega$ is a constant. The upstream reservoir viscosity $\mu_{0}$ is related to the Knudsen number there (based on ' $a$ '), $k n_{0}$, by

$$
\mu_{0}=\frac{1}{2} \rho_{0} a \mathrm{Kn}_{0}\left(8 \mathrm{RT} \mathrm{T}_{0} / \pi\right)^{1 / 2}
$$

giving

$$
\nu=\frac{\left(2 R T_{0}\right)^{1 / 2}}{2 a k n_{0}}\left(\frac{T}{T_{0}}\right)^{1-\omega}\left(\frac{\rho}{\rho_{0}}\right)
$$

It follows that the local knudsen number, $\mathrm{Kn}$, is given by

$$
K n=k n_{0}\left(\frac{T}{T_{0}}\right)^{\omega-1 / 2}\left(\frac{\rho_{0}}{\rho}\right)
$$


Instead of a Cartesian coordinate system for the velocity vector $\xi_{1}$, we introduce a polar coordinate system $\left(\xi_{p}, \theta, \xi_{z}\right)$ which is made nondimensional by scaling with $\left(2 R_{0}\right)^{1 / 2}$. We scale all length dimensions by ' $a$ '. Then the length of the slot is ' $\ell$ ' and its half width 1 . We denote by $(x, y, z)$ the scaled values of $(x 1, y 1, z 1)$. Thus,

$$
\begin{aligned}
& \xi_{z}=\xi_{z 1} /\left(2 R_{0}\right)^{l / 2} \\
& \xi_{p}{ }^{2}=\left(\xi_{x 1}{ }^{2}+\xi_{y 1}{ }^{2}\right) / 2 R T_{0}
\end{aligned}
$$

and

$$
\theta=\operatorname{Tan}^{-1}\left(\xi_{y 1} / \xi_{x 1}\right)
$$

Finally, we define

$$
F_{1}=\frac{\rho_{0}}{m}\left(2 \pi R T_{0}\right)^{-3 / 2} f\left(\xi_{p}, \theta, \xi_{z} ; x, y\right) .
$$

Then the BGK equation and boundary conditions are as follows:

$$
\xi_{p}\left(\cos \theta \frac{\partial f}{\partial x}+\sin \theta \frac{\partial f}{\partial y}\right)=\frac{1}{2 K n_{0}}\left(\frac{\rho}{\rho_{0}}\right)\left(\frac{T}{T_{0}}\right)^{1-\omega}\left(f_{0}-f\right),
$$

where

$$
f_{0}=\left(\frac{\rho}{\rho_{0}}\right)\left(\frac{T_{0}}{T}\right)^{3 / 2} \exp \left\{-B \frac{T_{0}}{T}\right\}
$$

and

$$
B=\left[\xi_{z}{ }^{2}+\xi_{p}{ }^{2}+\left(u^{2}+v^{2}\right) / 2 R T_{0}-2(u \cos \theta+v \sin \theta) \xi_{p} /\left(2 R T_{0}\right)^{1 / 2}\right]
$$

with, 


$$
\begin{aligned}
& f=\exp \left(-\xi_{p}{ }^{2}-\xi_{z}{ }^{2}\right) \text { at } x=0,-1<y<1,-\frac{\pi}{2}<\theta<\frac{\pi}{2}, \\
& f=\alpha \exp \left(-\xi_{p}{ }^{2}-\xi_{z}{ }^{2}\right) \text { at } x=\ell,-1<y<1, \frac{\pi}{2} \leqslant \theta<\frac{3 \pi}{2}, \\
& v=0 \text {, i.e., } \iiint d \xi_{p} d \xi_{z} d \theta \xi_{p}{ }^{2} \sin \theta f=0
\end{aligned}
$$

and

$$
\begin{array}{r}
f=\left(\frac{\rho}{\rho_{0}}\right) \exp \left(-\xi_{p}{ }^{2}-\xi_{z}{ }^{2}\right) \text { at } y=1,0<x<\ell \\
\text { for } \pi<\theta<2 \pi
\end{array}
$$

and

$$
f\left(\xi_{p}, \theta, \xi_{z} ; x, 0\right)=f\left(\xi_{p},-\theta, \xi_{z} ; x,-0\right) \text { for } 0<x<\ell .
$$

The macroscopic quantities are now defined as follows:

$$
\begin{aligned}
& \frac{\rho}{\rho_{0}}=\pi^{-3 / 2} \iiint d \xi_{p} d \theta d \xi_{z} \xi_{p} f, \\
& (\rho u, \rho v) / \rho_{0}\left(2 R T_{0}\right)^{1 / 2}=\pi^{-3 / 2} \iiint d \xi_{p} d \theta d \xi_{z}(\cos \theta, \sin \theta) \xi_{p}{ }^{2} f, \\
& \frac{T}{T_{0}}=\pi^{-3 / 2} \iiint d \xi_{p} d \theta d \xi_{z} \xi_{p} B f \text {, } \\
& \left(P_{x x}, P_{y y}, P_{x y}\right) / p_{0}=\pi^{-3 / 2} \iiint d \xi_{p} d \theta d \xi_{z} \xi_{p}{ }^{3} f \\
& \text { - }\left(\cos ^{2} \theta, \sin ^{2} \theta, \cos \theta \sin \theta\right)
\end{aligned}
$$

of great importance is the modified distribution function-$g\left(\xi_{p}, \theta ; x, y\right)$, defined as

$$
g\left(\xi_{p}, \theta ; x, y\right)=\frac{1}{(\pi)^{1 / 2}} \int_{-\infty}^{\infty} d \xi_{z} f\left(\xi_{p}, \theta, \xi_{z} ; x, y\right) .
$$

Physically, the modified velocity distribution function $g\left(x, y ; \xi_{p}, \theta\right)$ is defined to be the number of molecules per unit volume of the $(x, y)$ space and the $\left(\xi_{p}, \theta\right)$ space, scaled by the number of 
molecules per unit volume of the $(x, y)$ space and $\left(\xi_{p}, \theta\right)$ space, upstream of the slot.

The governing equation for $g$ is obtained by integrating the BGK equation for $f$ with respect to $\xi_{z}$ to give,

$$
\xi_{p}\left(\cos \theta \frac{\partial g}{\partial x}+\sin \theta \frac{\partial g}{\partial y}\right)=\frac{1}{2 K n_{0}} \frac{\rho}{\rho_{0}}\left(\frac{T}{T_{0}}\right)^{1-\omega}\left(g_{0}-g\right),
$$

where

$$
g_{0}=\frac{\rho}{\rho_{0}}\left(\frac{T_{0}}{T}\right)^{3 / 2} \exp \left(-\frac{T_{0}}{T} B\right)
$$

The boundary conditions in terms of $g$ are,

$$
\begin{aligned}
& g=\exp \left(-\xi_{p}{ }^{2}\right) \text { at } x=0,-1<y<1,-\frac{\pi}{2}<\theta<\frac{\pi}{2}, \\
& g=\alpha \exp \left(-\xi_{p}{ }^{2}\right) \text { at } x=\ell,-1<y<1, \frac{\pi}{2}<\theta<\frac{3 \pi}{2}, \\
& v=0 \text {, i.e., } \iint d \xi_{p} d \theta \xi_{p}{ }^{2} \sin \theta g=0
\end{aligned}
$$

and

$$
g=\frac{\rho}{\rho_{0}} \exp \left(-\xi_{p}{ }^{2}\right) \text { at } y=1,0<x<\ell \text { for } \pi \leqslant \theta \leqslant 2 \pi
$$

and

$$
g\left(\xi_{p}, \theta, x, 0\right)=g\left(\xi_{p},-\theta, x,-0\right) \text { for } 0<x<\ell .
$$

The following macroscopic quantities are definable in terms of the moments of $\mathrm{g}$ : 


$$
\begin{aligned}
& \frac{\rho}{\rho_{0}}=\iint d \xi_{p} d \theta \xi_{p} g \quad, \\
& \left.(\rho u, \rho v) / \rho_{0}\left(2 R T_{0}\right)^{1 / 2}=\iint d \xi_{p} d \theta \xi_{p}{ }^{2} g(\cos \theta, \sin \theta)\right) \\
& \left(P_{x x}, P_{y y}, P_{x y}\right) / p_{0}=\iint d \xi_{p} d \theta \xi_{p}{ }^{3} g\left(\cos ^{2} \theta, \sin ^{2} \theta, \cos \theta \sin \theta\right)
\end{aligned}
$$

It is evident that the temperature $T$ cannot be defined in terms of $\mathrm{g}$. 


\subsection{MOMENT METHOD OF SOLUTION}

\subsection{Formulation}

Consider any arbitrary point $(x, y)$ in the slot: A discontinuous velocity distribution, made up of four parts-- $f_{1}, f_{2}$, $f_{3}$ and $f_{4}$, is assumed at that point and is constituted thus:

$$
\begin{aligned}
& f=f_{1} \text { for } 0 \leqslant \xi_{p} \leqslant \infty \text { and } 0 \leqslant \theta \leqslant \pi / 2, \\
& f=f_{2} \text { for } 0 \leqslant \xi_{p} \leqslant \infty \text { and } \pi / 2 \leqslant \theta \leqslant \pi, \\
& f=f_{3} \text { for } 0 \leqslant \xi_{p} \leqslant \infty \text { and } \pi \leqslant \theta \leqslant 3 \pi / 2
\end{aligned}
$$

and

$$
f=f_{4} \text { for } 0 \leqslant \xi_{p} \leqslant \infty \text { and } 3 \pi / 2 \leqslant \theta \leqslant 2 \pi \text {. }
$$

The $f_{i}$ 's take the form

$$
f_{i}=\frac{\rho_{j}(x, y)}{\rho_{0}} \exp \left(-\xi_{p}{ }^{2}-\xi_{z}{ }^{2}\right) \text { with } i=1,2,3,4
$$

The $\rho_{i}$ 's are unknown functions of $(x, y)$. Any moment of $f$ can be calculated in terms of the $\rho_{i}^{\prime} s$. Thus the moment $\langle\phi\rangle$ of $f$ with respect to a function $\phi\left(\xi_{p}, \theta, \xi_{z}\right)$ is given by,

$$
\begin{aligned}
\langle\phi\rangle= & \int_{-\infty}^{\infty} d \xi_{z} \int_{0}^{\infty} d \xi_{p} \xi_{p}\left(\int_{0}^{\pi / 2} f_{1} \phi d \theta+\int_{\pi / 2}^{\pi} f_{2} \phi d \theta\right. \\
& \left.+\int_{\pi}^{3 \pi / 2} f_{3} \phi d \theta+\int_{3 \pi / 2}^{2 \pi} f_{4} \phi d \theta\right) .
\end{aligned}
$$

For example, 


$$
\begin{aligned}
& \frac{\rho}{\rho_{0}}=\left(\rho_{1}+\rho_{2}+\rho_{3}+\rho_{4}\right) / 4 \\
& \frac{\rho u}{\left(2 R T_{0}\right)}=\left(\rho_{1}-\rho_{2}-\rho_{3}+\rho_{4}\right) / 4 \pi^{1 / 2}
\end{aligned}
$$

The problem is hence resolved if the unknown functions $\rho_{i} \quad(i=1,2,3,4)$ are found. These four functions are determined by taking moments of the Maxwell Boltzmann equation. For any quantity $\phi\left(\xi_{p}, \theta, \xi_{z}\right)$ :

$$
\begin{aligned}
& \frac{\partial}{\partial x}\left(\iiint d \xi_{z} d \xi_{p} d \theta \xi_{p}{ }^{2} \cos \theta \phi f\right) \\
& +\frac{\partial}{\partial y}\left(\iiint d \xi_{z} d \xi_{p} d \theta \xi_{p}^{2} \sin \theta \phi f\right) \\
& =\frac{\rho}{\rho_{0}}\left(\frac{T}{T_{0}}\right)^{1-\omega} \frac{1}{2 K n_{0}} \iiint\left(f_{0}-f\right) \phi \xi_{p} d \xi_{p} d \theta d \xi_{z} \\
& =\Delta_{e}\langle\phi\rangle
\end{aligned}
$$

The $\rho_{i}(i=1$ to 4$)$ are replaced by $M_{00}, M_{01}, M_{10}$ and $M_{11}$ as unknowns, defined thus:

$$
\begin{aligned}
& M_{00}=\left(\rho_{1}+\rho_{2}+\rho_{3}+\rho_{4}\right) / 4 \\
& M_{01}=\left(\rho_{1}+\rho_{2}-\rho_{3}-\rho_{4}\right) / 4 \pi^{1 / 2} \\
& M_{10}=\left(\rho_{1}-\rho_{2}-\rho_{3}+\rho_{4}\right) / 4 \pi \\
& M_{11}=\left(\rho_{1}-\rho_{2}+\rho_{3}-\rho_{4}\right) / 4 \pi
\end{aligned}
$$

These new variables are noteworthy since," 


$$
\begin{aligned}
& M_{00}=\frac{\rho}{\rho_{0}}=\frac{1}{2} \frac{P_{x x}}{P_{0}}=\frac{1}{2} \frac{P_{y y}}{P_{0}}=\frac{1}{2} \frac{P_{z z}}{P_{0}} \\
& M_{10}=\rho u / \rho_{0}\left(2 R T_{0}\right)^{1 / 2} \\
& M_{01}=\rho v / \rho_{0}\left(2 R T_{0}\right)^{1 / 2}
\end{aligned}
$$

while

$$
M_{11}=\frac{P_{x y}}{2 p_{0}}
$$

The required equations for the four unknowns $M_{00}, M_{01}, M_{10}$ and $M_{11}$ are found by taking $\phi=m, m \xi_{p} \cos \theta, m \xi_{p} \sin \theta$ and $m \xi_{p}{ }^{2} \sin \theta \cos \theta$. The choice of these values for $\phi$ is dictated by considerations detailed earlier in Section $1: 2$. The equations obtained are as follows:

continuity equation --

$$
\frac{\partial M_{10}}{\partial x}+\frac{\partial M_{01}}{\partial y}=0
$$

$x$-momentum equation --

$$
\frac{1}{2} \frac{\partial M_{00}}{\partial x}+\frac{\partial M_{11}}{\partial y}=0
$$

$\therefore y$-momentum equation --

$$
\frac{\partial M_{11}}{\partial x}+\frac{1}{2} \frac{\partial M_{00}}{\partial y}=0
$$

while the shear stress equation is

$$
\frac{\partial M_{01}}{\partial x}+\frac{\partial M_{10}}{\partial y}=-\frac{(\pi)^{1 / 2} M_{00}}{K_{0}}\left(\frac{T}{T_{0}}\right)^{1-\omega}\left(M_{11}-M_{10} \cdot M_{01} / M_{00}\right),
$$

where

$$
\frac{T_{0}}{T_{0}}=1-\frac{2}{3}\left(M_{10}{ }^{2}+M_{01}^{2}\right) / M_{00}^{2}
$$


The boundary conditions expressed earlier in terms of $f$ can be expressed now in terms of $M_{00}, M_{01}, M_{10}$ and $M_{11}$. They are as follows:

At $x=0$,

$$
M_{00}+\pi^{1 / 2} M_{10}=1
$$

and

$$
\pi^{1 / 2} M_{11}+M_{01}=0
$$

At $x=\ell$,

$$
M_{00}-\pi^{1 / 2} M_{10}=
$$

and

$$
\pi^{1 / 2} M_{11}-M_{01}=0
$$

At $y=1$ and $y=0$,

$$
M_{01}=0
$$

and

$$
\pi^{1 / 2} M_{11}-M_{10}:=0
$$

The above system of equations is hyperbolic. The boundary conditions are, however, unorthodox since only two $\left(\rho_{1}\right.$, and $\left.\rho_{4}\right)$ of the four unknown quantities are specified at $x=0$; the other two $\left(\rho_{2}\right.$ and $\left.\rho_{3}\right)$ are specified at $x=\ell$. This is a boundary value problem, and to reduce it to a classical initial value problem, all four quantities $\rho_{i}$ have to be specified at an initial front, say $x=0$. However, the question arises: Does a unique solution exist for the above system of equations with those unorthodox boundary conditions? Using the work of Saranson (1962), 
it is verified that a unique solution does exist for all values of the parameters. The details are elaborated in Appendix A.

\subsection{Free Molecule Solution}

In the limit $K n_{0} \rightarrow \infty$, which corresponds to the free molecule flow, the above equations can be solved in terms of two unknown functions $G$ and $H$, giving

$$
\begin{aligned}
& M_{00}=G(x+y)+G(x-y) \\
& M_{11}=-\frac{1}{2}\{G(x+y)-G(x-y)\} \\
& M_{01}=H(x-y)-H(x+y) \\
& M_{10}=H(x+y)+H(x-y)
\end{aligned}
$$

In general the corners of the boundary are points of singularity. If we assume that at the corners of the boundary $(x=0$ and $y=0, y=1 ; x=\ell$ and $y=0, y=1)$ multiple boundary conditions can be simultaneously applied, then closed form solutions can be obtained for the $G$ and $H$ functions for any rational value of $\ell$. However, the expressions for $G$ and $H$ for any rational value of $\ell$ are considerably complicated. Only integer values of $\ell$ are considered and the results for $G$ and $H$ are tabulated in Appendix B. An interesting feature of these results is that the solutions differ depending on whether $\ell$ is an even integer or an odd integer. Of particular interest is $M_{10}$, which is the mass flux ou scaled by $\left(2 R_{0}\right)^{1 / 2} \rho_{0}$. When $\ell$ is an even integer,

$$
M_{10}=\frac{(1-\alpha)}{2 \pi^{1 / 2}[1+(\ell / \pi)]}
$$


When $\ell$ is an odd integer,

$$
M_{10}=\frac{(1-\alpha)}{2 \pi^{1 / 2}\left(1+\frac{\ell}{\pi}-\frac{1}{\pi(\ell+2)}\right)}
$$

Both the above expressions are valid for all values of $x$ and $y$ in the slot. Since $M_{10}$ is a constant everywhere in the slot, hence $2 M_{10}$ gives the total mass flux through the slot scaled by $\rho_{0}\left(2 R T_{0}\right)^{1 / 2}$ a.

A discrepancy in the two expressions is evident. For large lengths, it is negligible, but becomes significant for shorter lengths. In Fig. 4 a plot of the mass flux through the slot vs. $\ell$ has been made and is compared with the accurate numerical solution of Reynolds and Ri.chey. Our solution for the mass flux is lower compared to Reynolds and Richey's solution. This error, for the range 1 to 12 for $\ell$; increases as $\ell$ : increases, being as much as $35 \%$ for $\ell=12$. In fact, if our calculations were continued for $\&$ greater than 12, the errors should get progressively worse. Also the difference in expressions for even and odd lengths is brought out as ripples in the curve (see Fig. 4).: This ripple, however, dies out as the length $l$ increases beyond $l:=6$. The inevitable conclusion is that the solution is significant only for $\ell$ between 6 and 12; beyond $\ell=12$, érrors involved in the calculated value of the mass flux get very large as $\ell$ increases.

\subsection{Transition Flow Calculations}

The system of hyperbolic equations is solved by the method of characteristics. 
The characteristics are given by:

$$
\frac{d y}{d x}= \pm 1
$$

The compatability conditions along the characteristics

are:

$$
\begin{aligned}
& \frac{d y}{d x} \frac{d M_{00}}{d x}+2 \frac{d M_{11}}{d x}=0 \\
& \frac{d y}{d x} \frac{d M_{10}}{d x}+\frac{d M_{01}}{d x}+\frac{\pi^{1 / 2}}{K n_{0}}\left(\frac{T}{T_{0}}\right)^{1-\omega}\left(M_{00} M_{11}-M_{01} M_{10}\right)=0
\end{aligned}
$$

The grid points are taken as shown in Fig. 2. NN grid points are taken at the entrance, spaced at a distance 1/NN from each other. The grid points immediately adjacent to the boundaries (the centerline and the wall) are at a distance 1/2NN from the boundaries. The two characteristics $d y / d x= \pm 1$ pass from each grid point, forming a grid pattern, as shown in Fig. 2. The length $\ell$ is always so chosen that $2 \ell N N$ is an even integer. Integrating the above equations and expressing the results in a finite difference form, we get the following expression for any three points $A, B$ and $C$ (see Fig. 2):

Along $\frac{d y}{d x}=1$,

$$
M_{00}(A)+2 M_{11}(A)=M_{00}(B)+2 M_{11}(B),
$$

and

$$
\begin{aligned}
M_{10}(A)+M_{01}(A) & =M_{10}(B)+M_{01}(B) \\
& -\frac{\Delta x \pi \pi^{1 / 2}}{K_{0}}\left(\left\{\left(M_{00} M_{11}-M_{01} M_{10}\right)\left(\frac{T}{T_{0}}\right)^{1-\omega_{\}}}{ }_{A}\right.\right. \\
& +\left\{\left(M_{00} M_{11}-M_{01} M_{10}\right)\left(\frac{T}{T_{0}}\right)^{1-\omega_{3}}\right)_{B}
\end{aligned}
$$


Along $\frac{d y}{d x}=-1$,

$$
M_{00}(A)-2 M_{11}(A)=M_{00}(C)-2 M_{11}(C)
$$

and

$$
-M_{10}(A)+M_{01}(A)=-M_{10}(C)+M_{01}(C)
$$

with

$$
\begin{aligned}
& -\frac{\Delta x \pi^{1 / 2}}{K n_{0}}\left(\left\{\left(M_{00} M_{11}-M_{01} M_{10}\right)\left(\frac{T}{T_{0}}\right)^{1-\omega_{j}} A\right.\right. \\
& +\left\{\left(M_{00} M_{11}-M_{01} M_{10}\right)\left(\frac{T}{T_{0}}\right)^{1-\omega_{3}}\right)_{C},
\end{aligned}
$$

$$
\frac{T}{T_{0}}=1-\frac{2}{3} \frac{\left(M_{10}{ }^{2}+M_{01}{ }^{2}\right)}{M_{00}{ }^{2}}
$$

and

$$
\Delta x=1 / 2 N N
$$

Thus knowing the various $M^{\prime} s$ at. $B$ and $C$, the $M^{\prime} s$ at $A$ can be immediately obtained. Thus if all the M's are known at $x=0$, then the M's at all other grid points can be obtained with ease. When $\omega \neq 1$, at each point an iteration is involved to calculate $M_{10}$ and $M_{01}$. When $\omega=1$, an iteration is involved only to calculate $M_{10}$ at the wall. Define

$$
\begin{aligned}
& P^{\prime}=M_{00}-\pi^{1 / 2} M_{10} \\
& Q^{\prime}=\pi^{1 / 2} M_{11}-M_{01} \\
& R^{\prime}=M_{00}+\pi^{1 / 2} M_{10} \\
& S^{\prime}=\pi^{1 / 2} \cdot M_{11}+M_{01}
\end{aligned}
$$


A survey of the boundary conditions at $x=0$ reveals that $R^{\prime}=1, S^{\prime}=0$. No values for $P^{\prime}$ and $Q^{\prime}$ are available at $x=0$ to proceed with the initial value computation detailed above $\left(P^{\prime}\right.$ and $Q^{\prime}$ are available only at $x=\ell$, where $P^{\prime}=\alpha$, $\left.Q^{\prime}=0\right)$. However, an iteration procedure detailed in Appendix $C$ can be followed to arrive at the right starting values which would give $P^{\prime}=\alpha, Q^{\prime}=0$ at $x=\ell$. For the range of $\alpha, K n_{0}$ and $\ell$ considered, 12 was the maximum number of such iterations required.

A Fortran program was written to solve the system of algebraic equations. The following are the parameters in the problem: $\omega, \ell, N N, K n_{0}$ and $\alpha$. For the range of parameters considered, it was found that $T / T_{0}$ differed from unity only by $0\left(10^{-3}\right)$. Because of this weak influence of $T / T_{0}$, it was decided to keep $\omega=1$ and eliminate the iteration, except at the wall. For $k n_{0} \geqslant 1$, NN could be kept at a nominal value--between 3 and 5 . However, when $K n_{0}<1$, if $N N$ was kept too sma11, the iteration scheme for $M_{10}$ at the wall did not converge. The following criteria were evolved for $\mathrm{NN}$ (for $K n_{0}<1$ ) so that the iteration scheme for $M_{10}$ at the wall would be convergent:

$$
\mathrm{NN} K \mathrm{n}_{0}>(\pi)^{-1 / 2}-0.25 \alpha
$$

$\mathrm{Kn}$ o was given the values 5,1 and 0.5 , while $\ell$ had values 1 , $2,4,8$ and 12 .

The practice evolved in the computation was to keep $\ell$ and $\alpha$ fixed and vary $k n_{0}--$ starting with $k n_{0}=5$ and decreasing it to $K n_{0}=0.5$. To hasten the initial value iteration process, 
values obtained at $x=0$ for $P^{i}$ and $Q^{\prime}$ at the previous $k n_{0}$ were used for the next $k n_{0}$. 


\subsection{DISCRETE VELOCITY ORDINATE METHOD OF SOLUTION}

The discrete velocity ordinate method of solution takes cognizance of the fact that the distribution function (modified or otherwise) is a function of both space and velocity variables. However, in this method only certain discrete values of the velocity are considered and the distribution function (modified or otherwise) is evaluated at these discrete values of the velocity.

\subsection{Formulation}

In this method, the modified distribution function $g\left(\xi_{p}, \theta ; x, y\right)$ is the fundamental quantity that is evaluated. The function $g$, defined by (2.7), is governed by Eq. (2.6) with the boundary conditions (2.10).

Once $g$ is known, most moments, except for $T / T_{0}$, can be immediately evaluated--see Eqs. (2.10). A survey of the moment solution shows that $\left(T / T_{0}\right)$ varies from unity by less than $0.5 \%$ for the slot lengths considered. In consequence $\left(T / T_{0}\right)$ is set identically equal to unity. Thus, Eq. (2.8) has the simplified form,

$$
\xi_{p}\left(\cos \theta \frac{\partial g}{\partial x}+\sin \theta \frac{\partial g}{\partial y}\right)=\frac{\rho}{\rho_{0} 2 K n_{0}}\left(g_{0}-g\right)
$$

where

$$
\begin{aligned}
& g_{0}=\frac{\rho}{\rho_{0}} \exp \left\{-\left(\xi_{p} \cos \theta-u^{\prime}\right)^{2}-\left(\xi_{p} \sin \theta-v^{\prime}\right)^{2}\right\}, \\
& u^{\prime}=u /\left(2 R T_{p}\right)^{1 / 2} \text { and } v^{\prime}=v /\left(2 R T_{0}\right)^{1 / 2} .
\end{aligned}
$$

The boundary conditions (2.9) remain the same.

Formally, $g$ is a function of four independent, continuous variables, $x, y, \theta$ and $\xi_{p}$. However, the governing 
equation for $g,(4.1)$, has no derivatives with respect to either $\xi_{p}$ or $\theta$. Hence, in accord with the philosophy of the discrete ordinate method, $g$ is considered only at certain discrete values of $\xi_{p}$ and $\theta$. The choice of these values is strongly motivated by the physics of the problem.

Considering the present method as a natural extension of the moment method, the eight discrete values of $\theta$ are given by $\theta=\frac{M \pi}{4}$ with $M=0$ to 7 . The choice of the discrete values of $\xi_{p}$ is dictated by the consideration that our final interest is not in $g$ itself but in the moments of g--most notably the mass flux ou along the slot. For a fixed $\theta$, the free molecular and continuum functional form for $g$ is,

$$
\exp \left\{-\left(\xi_{p} \cos \theta-u^{\prime}\right)^{2}-\left(\xi_{p} \sin \theta-v^{\prime}\right)^{2}\right\} \quad .
$$

It seems reasonable to choose a quadrature formula that will be accurate for this form of $g$. As $u^{\prime}$ and $v^{\prime}$ are usually $\ll 1$, we linearize accordingly. The evaluation of $\rho, \rho u, \rho v$ and $P_{i j}$ $(i, j=x, y)$ involves integrals, with respect to $\xi_{p}$, of the form $\int_{0}^{\infty} d \xi_{p} \xi_{p} e^{-\xi_{p}^{2}} h_{1}\left(\xi_{p}\right)$

where $h_{7}\left(\xi_{p}\right)$ is a polynomial of degree 3 or less. We use a Gaussian quadrature formula for the evaluation of such integrals. Then the two discrete values of $\xi_{p}$ chosen are the Gaussian roots corresponding to the above integrals, denoted by $\xi_{a}$ and $\xi_{b}$. Let the corresponding Gaussian weights be denoted by $w_{a}$ and $w_{b}$. The numerical values of $\xi_{a}, \xi_{b}, w_{a}$ and $w_{b}$ are evaluated in Appendix D. 
The moments of $g$, besides being the quantities of primary interest, are, as seen from Eq. (4.1), involved in the solution of $g$ itself. These moments involve integration of $g$ with respect to both $\xi_{p}$ and $\theta$. As mentioned earlier, the integration with respect to $\xi_{p}$ is done using the Gaussian quadrature formulae. This is done for all values of $\mathrm{Kn}_{0}$, and it is anticipated that the accuracy of such a scheme would increase as $\mathrm{Kn}_{0} \rightarrow 0$ and $\infty$ when the speed distribution will be proportional to $\exp \left(-\xi_{\mathrm{p}}{ }^{2}\right)$. As for the integration of $g$ with respect to $\theta$, there are some important aspects to the flow that have to be considered. When the flow everywhere is close to continuum, $g$ varies continuously with respect to $\theta$. As the flow tends further and further from continuum, $g$ varies more and more steeply with respect to $\theta$. Finally, in the limit of free molecule flow, $g$ varies discontinuously with respect to $\theta$. To see this, consider an arbitrary point $(x, y)$ in the slot. Then the distribution function, $\mathrm{g}$ in the solid angles subtended by the entrance and the exit are $\exp \left(-\xi_{p}{ }^{2}\right)$ and $\alpha \exp \left(-\xi_{p}{ }^{2}\right)$, respectively. The number flux from the wall varies almost linearly along the length of the slot (see Reynolds and Richey, 1967). Translating this, the distribution function of the particles coming from the wall is close to the form $\{a(x, y)+$ $\cot \theta b(x, y)\} \exp \left(-\xi_{p}{ }^{2}\right)$, where. ' $a$ '. and ' $b$ ' are functions of $(x, y)$ and $\theta$ lies in the solid angle subtended by the wall at $(x, y)$.

First, a simple trapezoidal rule is used for integrating over the $\theta$ space, using the eight discrete values in the $\theta$.space. 
This scheme is very good when the $g$ varies slowly with respect to $\theta$. However, as the flow tends to the free molecule regime, $\mathrm{g}$ varies steeply and finally discontinuously with respect to $\theta$. Here this method of integration is inadequate.

The second mode of $\theta$ integration allows for the discontinuous form of $\mathrm{g}$ (with respect to $\theta$ ). In the solid angle subtended by both the entrance and the exit, the trapezoidal integration is used. In each of these solid angles, 1 to 3 discrete ordinates can lie in their span for each of the discrete values of $\xi_{p}$. In the solid angle subtended by each of the walls, the integration is done assuming that $g$ varies with $\theta$ as $\cot \theta$. In each of the solid angles subtended by the wall, for each discrete ordinate of $\xi_{p}, 2$ to 3 discrete ordinates in $\theta$ can lie in their span. Thus, if there are two such ordinates we assume that

$$
g\left(x, y ; \xi_{p}, \theta\right)=\left\{a\left(x, y ; \xi_{p}\right)+b\left(x, y ; \xi_{p}\right) \cot \theta\right\} \exp \left(-\xi_{p}{ }^{2}\right)
$$

and evaluate the two unknown functions $a\left(x, y ; \xi_{p}\right)$ and $b\left(x, y ; \xi_{p}\right)$ from the known values of $g$ for the two $\theta$ ordinates, for each speed $\xi_{p}$. If, however, 3 discrete ordinates 1 ie in the solid angle, assume that

$$
\begin{gathered}
g\left(x, y ; \xi_{p}, \theta\right)=\left\{a\left(x, y ; \xi_{p}\right)+b\left(x, y ; \xi_{p}\right) \cot \theta+c\left(x, y ; \xi_{p}\right)\right. \\
\left(\frac{k \pi}{2}-\theta\right)^{2} \exp \left(-\xi_{p}{ }^{2}\right),
\end{gathered}
$$

where $k=3,1$ for the top wall and bottom wall, respectively. The unknown functions $a, b, c$ are evaluated from the known values 
of $g$ at the three $\theta$ ordinate values (for each speed $\xi_{p}$ ). This method of integration is done not only for the free molecule flow, but also continued into the transition regime.

As emphasized earlier, the trapezoidal integration is increasingly accurate as the flow tends away from the free molecule regime. The $\cot \theta$ integration is certainly accurate in the free molecule regime; for it to be meaningful in the transition regime, the results obtained by this method have to tend to the results obtained by the trapezoidal integration.

Let $Q\left(\xi_{p}, \theta\right)=Q_{1}\left(\xi_{p}\right) Q_{2}(\theta)$ be a separable function whose moment (with respect to $g$ ) is desired. Thus, by the trapezoidal integration in $\theta$,

$$
\begin{aligned}
& \int_{0}^{\infty} \int_{0}^{2 \pi} d \xi_{p} d \theta \xi_{p} Q\left(\xi_{p}, \theta\right) g\left(x, y ; \xi_{p}, \theta\right) \\
& \quad=\sum_{i=a}^{b} \sum_{n=0}^{7} W_{i} Q_{j}\left(\xi_{j}\right) \exp \left(\xi_{i}{ }^{2}\right) g\left(x, y ; \xi_{i}, \frac{n \pi}{4}\right) Q_{2}\left(\frac{n \pi}{4}\right)
\end{aligned}
$$

By the $\cot \theta$ integration scheme for $\theta$,

$$
\begin{aligned}
& \int_{0}^{\infty} \int_{0}^{2 \pi} d \xi_{p} d \theta \xi_{p} Q\left(\xi_{p}, \theta\right) g\left(x, y ; \xi_{p}, \theta\right) \\
& =\sum_{i=a}^{b} W_{i} Q_{1}\left(\xi_{j}\right) \exp \left(\xi_{j}{ }^{2}\right) \int_{0}^{2 \pi} Q_{2}(\theta) g\left(x, y ; \xi_{p}, \theta\right) d \theta \\
& =\sum_{i=a}^{b} W_{i} Q_{j}\left(\xi_{j}\right) \exp \left(\xi_{j}{ }^{2}\right)\left\{\int_{\theta_{0}}^{\theta} d \theta Q_{2}(\theta) g\left(x, y ; \xi_{j}, \theta\right)\right. \\
& +\int_{\theta}^{\theta} d \theta Q_{2}(\theta)\left[a_{1}\left(x, y ; \xi_{j}\right)+b_{j}\left(x, y ; \xi_{j}\right) \cot \theta+\right.
\end{aligned}
$$




$$
\begin{aligned}
& \left.+c_{1}\left(x, y ; \xi_{j}\right)\left(\frac{\pi}{2}-\theta\right)^{2}\right]+\int_{\theta_{2}}^{\theta_{3}} d \theta Q_{2}(\theta) g\left(x, y ; \xi_{j}, \theta\right) \\
& +\int_{\theta_{3}}^{\theta_{4}} d \theta Q_{2}(\theta)\left[a_{2}\left(x, y ; \xi_{j}\right)+b_{2}\left(x, y ; \xi_{j}\right) \cot \theta\right. \\
& \left.\left.+c_{2}\left(x, y ; \xi_{j}\right)\left(\frac{3 \pi}{2}-\theta\right)^{2}\right]\right\}
\end{aligned}
$$

where

$$
\begin{aligned}
& \theta_{0}=\operatorname{Tan}^{-1}\left(\frac{1-y}{x}\right) \\
& \theta_{1}=\operatorname{Tan}^{-1}\left(\frac{1+y}{x}\right) \\
& \theta_{2}=\pi-\operatorname{Tan}^{-1}\left(\frac{1+y}{\ell-x}\right) \\
& \theta_{3}=\pi+\operatorname{Tan}^{-1}\left(\frac{1-y}{\ell-x}\right) \\
& \theta_{4}=2 \pi-\theta_{0}
\end{aligned}
$$

The first and third terms in the \{\} bracket represent integration over the slot entrance and exit solid angles, and are evaluated by trapezoidal integration; the second and fourth terms represent integration over the solid angle of the bottom and top wa11. Substituting $Q\left(\xi_{p}, \theta\right)=1, \xi_{p} \cos \theta, \xi_{p} \sin \theta$, etc., we can easily evaluate $\rho, \rho u, \rho v$, etc., from both (4.3a) and (4.3b). The grid points are taken as shown in Fig. 3. NN grid points, equally spaced, are taken in the $y$ direction starting from the wall and ending at the centerline. The index $J$ is used for the grid points in the $y$ direction; with $j=1$ representing the wall grid point and $J=N N$ the centerline of the slot. In the $x$ direction, the grid points are equally spaced by a distance 
1/(NN-1); they start at the entrance and end at the exit. The index $I$ is used for the grid points in the $x$ direction with $I=1$, representing the grid points at the slot entrance and $I=N=(N N-1) 2+1$, the grid points at the slot exit. The total number of grid points to be considered equals $\mathrm{NN} \cdot \mathrm{N}$. The grid points at the slot entrance and exit could be considered either just inside or outside the slot. The boundary conditions at the slot entrance and exit are accordingly different.

Let us use the notation $g(I, J, K, n)$ to denote the value of $g$ at the grid point located at $(x, y)$ given by

$$
\begin{aligned}
& x=(I-1) /(N N-1) \\
& y=(J-1) /(N N-1) ;
\end{aligned}
$$

$K=1$ and 2 are the respective values used to denote $\xi_{\mathrm{p}}=\xi_{\mathrm{a}}$ and $\xi_{p}=\xi_{b}$, while $n$ can have the values 0 to 7 , and this is defined by $\theta=n \pi / 4$. Consider the case where the grid points at the slot exit and entrance are just outside the slot. Then the boundary conditions at the slot entrance and exit grid points are as follows: At the slot entrance $g(1, J ; K, n)=\exp \left(-\xi_{p}{ }^{2}\right)$, for $J=1$ to $N$, $K=1$ and 2 and $n=0,1,2,6$ and 7 . In addition, at the slot corner $(x=0, y=1)$, it is assumed that $g(1,1 ; K, 5)=\exp \left(-\xi_{p}{ }^{2}\right)$ for $K=1$ and 2. At the slot exit, $g(N, J ; K, n)=\alpha \exp \left(-\xi_{p}{ }^{2}\right)$ for $J=1$ to $N N, K=1$ and 2 , and $\mathrm{n}=2$ to 6 . Also at the slot corner $(x=1, y=1)$ it is assumed that $g(N, 1 ; K, 7)=\alpha \exp \left(-\xi_{p}{ }^{2}\right)$ for $k=1$ and 2 .

Consider the case where the grid points at the slot 
entrance and exit are taken just inside the slot. Then the boundary conditions at the slot entrance and exit grid points are as follows: At the slot entrance $g(1, J ; K, n)=\exp \left(-\xi_{p}{ }^{2}\right)$ for $J=2$ to $N N$, $K=1$ and 2 , and $n=0,1$ and 7 . At the wall, $J:=1, g(1, I ; K, n)$ $=\exp \left(-\xi_{p}^{2}\right)$ for $K=1$ and 2 and $n=0$ and 7 ; also the reflection from the wall is diffuse and $v=0$. At the centerline, $J=N N$, $g(1, N N ; K, n)=g(1, N N ; K, 8-n)$ for $K=1$ and $2, n=1,2,3$. At the slot exit $g(N, J ; K, n)=\alpha \exp \left(-\xi_{p}{ }^{2}\right)$ for $J=2$ to $N N, K=1$ and 2 , and $n=3,4$ and 5. At the wal1, $J=1, g(N, 1 ; K, n)=\alpha \exp \left(-\xi_{p}{ }^{2}\right)$ for $K=1$ and 2 , and $n=3$ and 4 ; also the reflection from the wall is diffuse and $v=0$. At the centerline, $J=N N, g(N, N N ; K, n)=$ $g(N, N N ; K, 8-n)$ for $K=1$ and $2, n=1,2$ and 3 .

In addition to the above two possible boundary conditions at the slot entrance and exit we have the following boundary conditions valid for $I=2$ to $(\mathrm{N}-1)$. At the wall, $\mathrm{J}=1, \mathrm{v}=0$ and the reflection from the wall is diffuse. At the centerline, $J=N N$, by symmetry about the $y$ axis, $g(I, N N ; K, n)=g(I, N N ; K, 8-n)$ for $K=1$ and 2 , and $n=1,2$ and 3 .

Numerical experiments with the above two possible sets of boundary conditions (at the slot entrance and exit grid points) were performed. The free molecule results obtained by assuming that the grid points are just outside the slot entrance and exit are much better (by comparison with the solutions of Reynolds and Richey). Henceforth it is assumed that the grid points at the slot entrance and exit are just outside the slot. 
Checking our list, we find that with the grid points at slot entrance and exit placed outside the slot, (6NN-4) quantities have to be assumed to specify $g(1, J ; K, n)$ completely for all values of $\mathrm{J}, K$ and $n$. In the case of free molecule flow (see Section 4.2) several simplifying features exist. Consequently, there is a rather dramatic decrease in the number of quantities to be assumed to specify $g(1, J ; K, n)$ completely for all $J, K$ and $n$.

At the wall we note that $g(I, 1 ; K, 0)$ and $g(I, 1 ; K, 5)$ are both discontinuous and have two values each. This is because of the two faced character of the distribution function $g$ at the wall, since there are essentially two totally different classes of molecules there--the molecules reflected from the wall and those coming into the wall.

\subsection{Free Molecule Limit Solution}

In the free molecule limit, i.e., $\mathrm{Kn}_{0} \rightarrow \infty$, Eq. (4.1) reduces to,

$$
\cos \theta \frac{\partial g}{\partial x}+\sin \theta \frac{\partial g}{\partial y}=0
$$

Integrating (4.4) along the characteristic direction, $d y / d x=$ $\tan \theta$, the solution of (4.4) is that $g$ is a constant along the characteristic direction $d y / d x=\tan \theta$, for all $\xi_{\mathrm{p}}$.

In terms of our grid points set and discrete velocity ordinates this can be interpreted as, 


$$
\begin{aligned}
& g(I+1, J ; K, 0)=g(I, J ; K, 0) \\
& g(I+1, J ; K, 1)=g(I, J+1 ; K, 1) \\
& g(I+1, J ; K, 2)=g(I+1, J-1 ; K, 2) \\
& g(I+1, J ; K, 3)=g(I, J-1 ; K, 3) \\
& g(I+1, J ; K, 4)=g(I, J ; K, 4) \\
& g(I+1, J ; K, 5)=g(I, J+1 ; K, 5) \\
& g(I+1, J: K, 6)=g(I+1, J+1 ; K, 6) \\
& g(I+1, J ; K, 7)=g(I, J-1 ; K, 7)
\end{aligned}
$$

for $I=1$ to $N-1, K=1$ and 2 and $J=1$ to $N N$.

To reduce the two point boundary value problem to an initial value problem, it is required that $g(1, J ; K, n)$ be completely known for $\mathrm{J}=1$ to $N N, K=1$ and 2 and $n=0$ to 7 . As discussed in an earlier section, in general (6NN-4) of these quantities have to be assumed. However, for this free molecule flow situation the distribution function is, for $\theta$ constant, directly proportional to $\exp \left(-\xi_{p}^{2}\right)$. Hence only $K=1$ has to be considered. Also, for $\theta=\pi$, i.e., $n=4$, the value is given by the value at $x 1=l a, i . e ., \quad I=N$. Hence we need to assume only $(2 \mathrm{NN}-2)$ quantities at $x \mathrm{~T}=0$, i.e., $I=1$. When $g(1, J ; K, n)$ has been assigned for $J=1$ to $N N$, $n=0$ to 7 and $k=1$, say, the values of $g(2, J ; K, n)$ can be 
computed as follows:

At the wall we have from $(4.4 a)$,

$$
g(2,1 ; K, 5)=g(1,2 ; K, 5)
$$

i.e., we know the distribution function for molecules leaving the wall at $\theta=5 \pi / 4$. By the condition of diffuse reflection,

$$
g(2,1 ; K, n)=g(2,1 ; K, 5) \text { for } n=4,6,7 \text { and } 0 \text {, }
$$

with the understanding that the values for $n=0$ and 4 represent the outgoing distribution function. We now have $g(2, J ; K, 6)=$ $\mathrm{g}(2, \mathrm{~J} ; \mathrm{K}, 2)$ for $\mathrm{J}=1$ to $\mathrm{NN}$ by symmetry (the number flux at the two walls is the same for the same $x$ value). Equations (4.4a) now serve to determine $g(2, J ; K, n)$ for all values of $J=2$ to $(N N-1)$, and $g(2, N N ; K, n)$ for $n=4,5,6$ and 7 , i.e., molecules crossing the centerline from $y \geqslant 0$. By the symmetry at the centerline we also have, $g(2, N N ; K, 1)=g(2, N N ; K, 7)$, i.e., $\theta=\pi / 4$ and $7 \pi / 4$ and $g(2, N N ; K, 3)=g(2, N N ; K, 5)$. The only remaining value is that for the wall and $\theta=3 \pi / 4$, i.e., $g(2,1 ; k, 3)$. While all previous relations are exact for free molecule flow we have to determine $g(2,1 ; K, 3)$ by the condition of zero normal mass flux at the wall. The accuracy of this calculation depends on the accuracy of the $\theta$ quadrature formula.

The above process can be repeated for all values of I up to N. At $I=N$ the calculated values of $g(I, J ; K, n)$ for $J=1$ to NN and $n=3$ and 5 have to be equal to $\alpha \exp \left(-\xi_{p}{ }^{2}\right)$. If they are not, an iteration scheme, similar to that detailed in 
Appendix $C$, can be used to obtain those values of $g(1, J ; K, n)$ for $J=1$ to $N N$ and $n=3$ and 4. which give $g(N, J ; K, n)=$ $\alpha \exp \left(-\xi_{p}{ }^{2}\right)$ for $J=1$ to $N N$ and $n=3$ and 5 . A total of (2NN-1) sweeps from $I=1$ to $N$ are required for each such iteration.

The solutions for $g\left(I, J ; \xi_{p}, \theta\right)$ were obtained by both the trapezoidal and $\cot \theta$ integration schemes. These solutions are different as the computational form for the boundary condition $\rho v=0$ at the wall is different by the two methods. The macroscopic quantities and the mass flux through the slot (by trapezoidal integration of ou across the slot section) were also obtained by both forms of $\theta$ integration. The only parameter varied is the slot length $\ell$, since the free molecule solution depends linearly on $\alpha$.

The mass flux through the slot obtained by the trapezoidal integration (with respect to $\theta$ ) agrees well with the results of Reynolds and Richey for small slot lengths. However, as the slot length increases there is a systematic overestimation of the mass flux with an error of $25 \%$ for a slot length of 12 . By the $\cot \theta$ integration, there is a spectacular agreement with the mass flux results of Reynolds and Richey with an error of less than $1 \%$ for a slot length up to 12 . This is not surprising as we took advantage of the linear wall flux dependence to pick our $\theta$ quadrature scheme.

Figure 4 displays the mass flux through the slot, as a function of slot length, by both the $\cot \theta$ and trapezoidal 
integrations. The slot lengths were considered over the range 1 to 12 .

\subsection{Transition Flow Calculations}

Here the entire equation (4.1), with associated boundary conditions for $\mathrm{g}$ has to be considered. Replacing the left hand side of Eq. (4.1) by the derivative along the characteristic direction $d y / d x=\tan \theta$, dividing by $\xi_{p}$ and introducing a quantity $L$,

$$
\frac{d g}{d s}=L=\frac{\rho}{\xi_{p} \rho_{0} 2 K n_{0}}\left(g_{0}-g\right)
$$

where $d x / d s=\cos \theta$ and $d y / d s=\sin \theta$. Expressing (4.5) in $a$ simple finite difference form, we have

$$
g\left(s+\Delta s ; \xi_{p}, \theta\right)=g\left(s ; \xi_{p}, \theta\right)+L\left(s, \xi_{p}, \theta\right) \Delta s,
$$

where $L$, the right hand side of Eq. (4.5), is evaluated at $s$. Equation (4.6) can be specialized for the discrete velocity ordinates values. Let us again use the notation $g(I, J ; K, n)$ to denote the value of $g$ at the grid point $(I, J)$ with $K=1$ and 2 representing $\xi_{p}=\xi_{a}$ and $\xi_{b}$, while $\theta=n \pi / 4$. Then, for our grid points, the following equations are valid:

$$
\begin{aligned}
& g(I+1, J ; K, 0)=g(I, J ; K, 0)+\frac{1}{(N N-1)} L(I, J ; K, 0) \\
& g(I+1, J ; K, I)=g(I, J+1 ; K, 1)+\frac{2^{1 / 2}}{(N N-I)} L(I, J+1 ; K, 1) \\
& g(I+1, J ; K, 2)=g(I+1, J-1 ; K, 2)-\frac{1}{(N N-1) L(I+1, J-1 ; K, 2)} \\
& g(I+1, J ; K, 3)=g(I, J-1 ; K, 3)-\frac{2^{1 / 2}}{(N N-1)} L(I, J-I ; K, 3) \\
& g(I+1, J ; K, 4)=g(I, J ; K, 4)-\frac{1}{(N N-1)} L(I, J ; K, 4)
\end{aligned}
$$




$$
\begin{aligned}
& g(I+1, J ; K, 5)=g(I, J+1 ; K, 5)-\frac{2^{1 / 2}}{(N N-1)} L(I, J+1 ; K, 5) \\
& g(I+1, J ; K, 6)=g(I+1, J-1 ; K, 6)+\frac{1}{(N N-1)} L(I+1, J-1 ; K, 6) \\
& g(I+1, J ; K, 7)=g(I, J-1 ; K, 7)+\frac{2^{1 / 2}}{(N N-1)} L(I, J-1 ; K, 7)
\end{aligned}
$$

where $I=1$ to $(N-1), J=1$ to $N N$ and $K=1$ and 2 .

For purposes of computation, $g(I, J ; K, 7)$ has to be completely specified at $I=1$ for $J=1$ to $N N, n=0$ to 7 and $K=1$ and 2. As detailed in Section (4.1), (6NN-4) quantities have to be assumed to fully specify $g(I, J ; K, n)$ at $I=1$ for all values of $J, K$ and $n$.

For the transition flow, there is no simple relation connecting $g(I, J ; K, n)$ for different values of $K$. Once again, for $I=2$ to $(N-1)$ and $K=1$ and 2 , both $g(I, 1 ; K, 0)$ and $g(I, 1 ; K, 4)$, i.e., the value of the distribution function at the wall for $\theta=0$ and $\pi$ is double valued. Also, we find that for $K=1$ and 2, $g(1, J ; K, 4)$, i.e., $\theta=\pi$ is not immediately known. Further, we find that $g(I, 1 ; K, 3)$ for $K=1$ and $I=2$ to $(N-1)$ have to be assumed. Thus in all $(6 N N+N-6)$ quantities have to be assumed in all to reduce the two point boundary value problem to an initial value problem. Therefore, the transition flow calculations are considerably more complicated than the free molecule flow calculations.

When $g(1, J ; K, n)$ has been assigned for $j=1$ to $N N$, $K=1$ and 2 and $n=0$ to 7 , the values of $g(2, J ; K, n)$ can be computed as follows: 
At the wall, using $(4.7)$, we can calculate $g(2,1 ; K, 5)$ in terms of $g(1,2 ; K, 5)$ for both $K=1$ and 2 , i.e., we know the distribution function for molecules leaving the wall at $\theta=5 \pi / 4$. By the condition of diffuse reflection,

$$
g(2,1 ; K, n)=g(2,1 ; K, 5) \text { for } n=0,4,6 \text { and } 7 \text { and } K=1 \text { and } 2 \text {. }
$$

The understanding here is that the values for $\mathrm{n}=0$ and 4 represent the outgoing distribution function. Further, using Eqs. (4.7), $g(2,1 ; K, n)$ can be calculated for $K=1$ and 2 and $n=0,1$ and 4.; the values here for $n=0$ and 4 are understood to represent the incoming distribution function. Since $g(2,1 ; 1,3)$, i.e., the value of $g$ at the wall for $\xi_{p}=\xi_{a}$ and $\theta=3 \pi / 4$, has been assumed, hence all values of $g(2,1 ; K, n)$ have been found except for $g(2,1 ; 2,3)$ and $g(2,1 ; K, 2)$ for $K=1$ and 2 . These quantities correspond to $\xi_{p}=\xi_{b}, \theta=3 \pi / 4$ and $\xi_{p}=\xi_{a}, \xi_{b}, \theta=\pi / 2$, respectively. As an initial guess for $g(2,1 ; K, 2)$, assume that $g(2,1 ; K, 2)=g(2,1 ; K, 6)$, i.e., $g$ is the same for $\theta=\pi / 2$ and $\theta=3 \pi / 2$, for $K=1$ and 2 . Then $g(2,1 ; 2,3)$ can be calculated using the boundary condition $\rho v=0$ at the wall. Thus $g(1, J ; K, n)$ is completely specified at the wall, $J=1$.

Using (4.7), $g(2, J ; K, n)$ can now be completely specified for $J=2$ to $(\mathrm{NN}-1), K=1$ and 2 and $n=0$ to 7 . At the centerline $J=N N, g(2, N N ; K, n)$ can be calculated for $K=1$ and 2 and $n=0,2,3,4,6$ and 7 . Symmetry conditions there also give,

$$
g(2, N N ; K, 5)=g(2, N N ; K, 3)
$$


and

$$
g(2, N N ; K, 1)=g(2, N N ; K, 7) \quad \text { for } K=1 \text { and } 2 .
$$

Further, symmetry conditions at $J=N N$ require that $g(2, N N ; K, 2)=$ $g(2, N N ; K, 4)$ for $K=1$ and 2 . If our calculations do not give this, it means that the assumed values of $g$ at the wall for $\theta=\pi / 2$ and $\xi_{p}=\xi_{a}$ and $\xi_{b}$, i.e., $g(2,1 ; K, 2)$ are not appropriate. An iteration scheme similar to that in Appendix $C$ can be followed to obtain accurate values of $g(2,1 ; K, 2)$ for $K=1$ and 2 which would give, to sufficient numerical accuracy, $g(2, N N ; K, 2)=$ $g(2, N N ; K, 6)$. Thus, $g(2, J ; K, n)$ can be completely calculated for all values of $J, K$ and $n$.

The above process of calculation used for $g(2, J ; K, n)$ can be repeated for all values of $I$ up to $N$. The check to see if the $(6 \mathrm{NN}-6+\mathrm{N})$ values that were assumed were the appropriate ones consists of two parts. First, since the reflection from the wall was assumed to be diffuse, it is required that

$$
g(I, 1 ; 1,5) \exp \left(\xi_{a}{ }^{2}\right)=g(I, 1 ; 2,5) \exp \left(\xi_{b}{ }^{2}\right) \text { for } I=2,(N-1)
$$

This gives $(\mathrm{N}-2)$ matching conditions. Further, it is required that $g(N, J ; K, n)=\alpha \exp \left(-\xi_{p}{ }^{2}\right)$ for $J=1, N N, K=1$ and 2 and $n=3,4$ and 5 . If these conditions are not satisfied an iteration scheme like that in Appendix $C$ can be used to get the $(6 \mathrm{NN}-4+\mathrm{N}-2)$ values required. For each such iteration $(6 \mathrm{NN}+\mathrm{N}-5)$ sweeps are required through the field $I=1$ to $I=N$.

Calculations were performed for $\ell=1,2,4,8,12, \alpha=0.8$, $0.5,0.1$ and $k n_{0}=5.0,1.0,0.5$. The procedure used was to keep 
the length $\ell$ constant first. Then $\alpha$ is systematically varied. However, for each $\alpha, \mathrm{Kn}_{0}$ is systematically varied from 5.0 to 0.5. The initial guess given for $k n_{0}=5.0$ is taken from the free molecule flow values. The initial guess for subsequent values of $\mathrm{Kn}_{0}$ is the calculated value at the previous $\mathrm{Kn}_{0}$. The $\theta$ integrations were performed by both the trapezoidal and $\cot \theta$ integrations. For the parameters considered $\left(K n_{0} \leqslant 5\right)$ the agreement by the two modes of $\theta$ integration is very good, though the computer time involved for the $\cot \theta$ integration is considerably more than for the trapezoidal integration. Also as the slot length is increased, the computer time consumed in the calculation increases as the square of the slot length. For a slot length of 12 a number of minutes of computer time (CDC 6400) are required. This could be pinpointed as one of the deficiencies of the method. A comparison of the solutions obtained here with the moment method shows a remarkably good agreement--the difference is less than $5 \%$ for $K n_{0}=0.5,1.0$ and 5.0. However, as $K n_{0}$ gets larger, a considerable difference in solutions is expected between the two methods. This is because the free molecule solutions by the two methods are so different. 


\subsection{DISCUSSION OF SOLUTIONS}

Solutions by both the moment and the discrete ordinate methods have been obtained for free molecule and transition flows. The latter method employed two forms of angular integration in the velocity space, the trapezoidal rule and the cotangent integration. The range of parameters considered is $\alpha=0.1,0.5$ and 0.8 , $\ell=1,2,4,8$ and 12 , and $k n_{0}=\infty, 5,1.0$ and 0.5 .

\subsection{Free Molecule Results}

The free molecule solutions are compared with the solutions of Reynolds and Richey (1967). The number flux from the wall by the moment method is shown in Figs. 7 and 8 for lengths $\&=4$ and 8 ; Figs. 5 and 6 show the same by the discrete ordinate method with both forms of integration. Surveying these figures, we find that the discrete ordinate method with the cotangent integration agrees closely with the linear wall flux variation obtained by the solution of Reynolds and Richey. This is not surprising, since we had specifically tailored our $\theta$ integration to a linear wall flux variation. The wall flux by the moment method and the discrete ordinate method with a trapezoidal integration seem to follow the overall linear variation of Reynolds and Richey but have step like variations. These steps correspond to sudden changes in the wall flux values which occur for even integer values of $x$, the coordinate along the slot axis. This is attributable to the fact that the only oblique angular direction considered in the velocity space is $\pi / 4$ with respect to the slot axis, and hence there is a certain periodicity over distances equal to the total slot width $(=2)$. Figure 4 
shows the mass flux through the slot by the various methods. The moment method underestimates the mass flux; the error continues to increase as the slot length increases. On the other hand, the discrete ordinate method with the trapezoidal integration overestimates the mass flux; the error continues to increase as the slot length increases. In both these cases, the poor results are due to no allowance being made for the discontinuity of the distribution function with respect to $\theta$ and also for the linear variation of the distribution function along the slot axis. The discrete ordinate method with a cotangent integration makes allowance for both these factors. Hence the mass flux computed here agrees very well with that by Reynolds and Richey.

\subsection{Transition Flow Results}

In the transition regime, for the values of $\mathrm{Kn}_{0}$ considered, the distribution function varies continuousiy with respect to $\theta$ (except at the walls). Hence the differences in the discrete ordinate solutions with trapezoidal and cotangent integrations is barely discernable. This can be seen, for example, in Fig. 9, where the wall flux values are plotted by the two methods for $\ell=1$, $\alpha=0.1$ and $K n_{0}=0.5$. These differences by the two methods should get less as the slot length increases. For the range of parameters considered the difference in the computed mass flux through the slot is less than $4 \%$. Figure 10 shows a plot of the number flux from the wall for $\ell=4, a=0.1, K n_{0}=5.0$ and 1.0 obtained by using the discrete ordinate method with trapezoidal integration. The number flux seems to vary slowly with respect to $x$ except close to even 
integer values of $x$. The significance of the even integer values of $x$ seems the same as for free molecule flow.

The moment method calculation in the transition regime yields results which compare very favorably with the discrete ordinate method solution. Figure 11 shows the plot of the number flux at the wall. The pattern seems to match that of the discrete ordinate solution, although the variations at even values of $x$ seem sharper. The significance of the even values of $x$ is the same as in the free molecule flow. Figure 12 shows the number flux from the wall vs. $x$, by the moment method for $l=12, \alpha=0.1$ and $K n_{0}=5.0,1.0$ and 0.5 . Remarkably, any periodicity with respect to even values of $x$ is absent. Further, the variation is almost linear. Also the values of the mass flux obtained by the moment method agree very we11 with that by the discrete ordinate method. For the range of parameters considered, the difference seems to increase as $k n_{0}$ increases, being a maximum of $2 \%$ for $K n_{0}=0.5$ and a maximum of $6 \%$ for $K n_{0}=5.0$. Further, the plane Poiseuille formula with slip boundary conditions (valid strictly only for an $\infty$ length slot) gives values for the mass flux through the slot that agree with the moment and discrete ordinate solutions for $k n_{0}=0.5$ and 1.0. The errors are less than $5 \%$. The remarkable feature here is that this result is valid for all lengths considered $\ell=1,2,4,8$ and 12 . However, we have to substitute the actual values of the average pressure at the slot entrance and exit rather than the values $p_{0}$ and $a p_{0}$ ? respectively.

For the parameters considered, the following interpolation formula is proposed for $\dot{m}$, the mass flux through the slot: 


$$
\begin{aligned}
\frac{\dot{m}}{\rho_{0} a\left(2 R T_{0}\right)^{T / 2}}= & \frac{1}{\ell}\left\{\frac{0.1331}{K n_{0}}(1+\alpha) T_{1}+2\left(T_{1}-\frac{K n_{0}}{8} \log S_{1}\right)\right. \\
& \left.+\frac{K n_{0}}{8 \sqrt{\pi}} \log S_{1} \log \left(\frac{\ell+\sqrt{\ell^{2}+4}}{2}\right)\right\},
\end{aligned}
$$

where

$$
\begin{aligned}
& T_{1}=\frac{(1-\alpha) l}{\ell+4.55-2.85 \exp \left\{0.21\left(K_{0}-5\right)\right\}} \\
& S_{1}=\frac{2 K n_{0}+8(1+\alpha+T)}{2 K n_{0}+8(7+\alpha-T)}
\end{aligned}
$$

The numerical values of $\dot{m}$ obtained by calculations differ from this formula by less than $5 \%$. The above formula is patterned after the formula proposed by Fryer (1966) for the mass flux through an infinite cylindrical tube in the transition regime. Crudely, in the expression for $\dot{m}$, the first term is the mass flux in the continuum regime, the second term the contribution due to slip at the boundary, and the last term the free molecule mass flux.

A systematic study on the effect of the number of grid points NN on the results obtained, both by the moment and discrete ordinate methods, was not possible due to the limitations on the computer time available. An intuitive prerequisite on the grid size is that it should be less than the mean free path. We also note that the integration of the differential equations of the moment and discrete ordinate methods is a first order scheme. Hence the accuracy of the solution increases as the grid size is decreased. A possible gauge to test the 'reasonableness' of the grid size is to check and see if the mass flux along the slot is conserved. The percentage deviations in the mass flux calculated can be taken as 
an index on the necessity for more grid points. A trapezoidal integration scheme is used to integrate $\rho \mathrm{across}$ the slot section to give the total mass flux through the slot.

A check of the moment solutions indicates that with NN between 3 and 5 , the maximum deviation varies, respectively, from 3 to $1 \%$. This is true both for the free molecule and transition flow calculations. For free molecule flows by the discrete ordinate method, with either trapezoidal or cotangent integration, the maximum deviation is 4 to $2 \%$ for NN between 3 and 6 , respectively. However, in the transition regime for NN between 3 and 5 , the maximum deviations were $9 \%$ to $4 \%$. In fact, NN was kept $=4$ for the transition regime calculations by the discrete ordinate method.

Figure 13 shows a plot of $Q_{f m} / Q_{\text {tran }}$ vs. $\left[K_{0} /(1+\alpha)\right]$ $\left[Q_{\text {cont }} / Q_{f m}\right]$; where $Q$ is the mass flux through the slot scaled by $\rho_{0} a\left(2 R T_{0}\right)^{1 / 2}$, and the subscripts $f m$, cont and tran denote the free molecule, continuum and transition flow regimes, respectively. This correlation is in the spirit of the work of Sherman (1963). However, a single curve for all values of the $\mathrm{Kn}_{0}$ could not be obtained. This was presumably because the mass flux through a finite length slot in the continuum limit was not known correctly. The $Q_{\text {cont }}$ used was obtained from the Poiseuille formula valid onty for infinite length slots.

Figure 14-1 shows the velocity profile (ou) across the slot section at the slot entrance, exit and midsection for $\ell=12$, $K n_{0}=5,1$ and 0.5 , and $\alpha=0.1$.

Comparing the moment and discrete ordinate methods, the 
discrete ordinate method seems to give good results for all the values of parameters considered. However, the amount of computing involved is many times that by the moment method (see below). The free molecule solution by the moment method is deficient, but the transition flow solution by the moment solution quickly merges with that by the discrete ordinate method. For the same number of grid points NN and slot length, the computation time for each iteration by the moment method varies as $\ell(N N)^{2}$, while by the discrete ordinate method as $\ell^{2}(N N)^{2}$. Further, it is found that in general the discrete ordinate method involves. a greater number of initial value iterations since it is very difficult to guess the values of the $g\left(I, 1 ; \xi_{a}, 3 \pi / 4\right)$ with any precision.

As $K n_{0}$ is decreased, the number of grid points has to be increased since physically it seems that the distance between the grid points has to be less than the mean free path. Thus both our methods of computation are going to be more and more time consuming (on the computer) as $\mathrm{Kn}_{0}$ is decreased. Further, as $\mathrm{Kn}_{0}$ is decreased, the iteration for the initial values becomes time consuming, but we also found that the iterations tend to diverge very quickly if the initial values given are not judiciously chosen. The only way to avoid divergence seems to be to decrease $k n_{0}$ slowly. Even this is not sufficient at times. The iterations have to be nursed along very carefully by decreasing $\varepsilon$ (see Appendix C) appropriately ( $\varepsilon$ is the increment in the initial values).

5.3 Improvements and Suggestions for Future Work As discussed earlier, the solutions for the wall flux, etc. 
seem to have a periodicity for even integer values of $x$. There is no reason to expect this in an actual flow. The reason it occurs in our solution is that $\pi / 4$ is the only oblique (with respect to the slot axis) discrete ordinate value of $\theta$ chosen. The obvious remedy is to take successively more numbers of oblique discrete values of $\theta$. Unfortunately, the computer time involved per iteration goes up very rapidly.

In view of the computer time consumed by discrete ordinate method calculations, it seems more appropriate to use a refined moment method, one preferably in the spirit of the work of Lees (1959). We do know the distribution function at any point in the slot for free molecule flow. In the solid angles subtended at the point by the slot entrance and exit, $f$, the normalized distribution function has the values $\exp \left(-\xi^{2}\right)$ and $\alpha \exp \left(-\xi^{2}\right)$. In the solid angle subtended by each wall, since the wall flux varies linearly with $x$, the normalized distribution function $f$ is given by $f=\left(a_{3}+b_{3} \cot \theta\right) \exp \left(-\xi^{2}\right)$, where $a_{3}$ and $b_{3}$ are known functions of $x$ and $y$. These distribution functions could be generalized to include unknown functions of the physical space. The advantage of this method is that it could represent the free molecule distribution very well. Also, in view of the good results of the discrete ordinate method with the cotangent integration, in the transition regime, it seems reasonable to expect favorable results towards the continuum regime. More important, the computer time required for computation would only be of the same order as that by the simple moment method. 


\subsection{CONCLUSIONS}

1. The moment method is used to obtain the free molecule and transition flow solutions. The range of parameters considered is: $\alpha=0.1,0.5$ and $0.8, \ell=1,2,4,8$ and 12 , and $k n_{0}=0.5$, $1.0,5.0, \infty$. The free molecule solution seems to be significant only in the range $l=6$. to $l=12$. However, the errors in the calculated value of the mass flux compared to the solution of Reynolds and Richey (1967) varies from 25\% for $\ell=6$ to $35 \%$ for $\ell=12$. The errors in fact get progressively worse as the slot length $\ell$ is increased beyond $\ell=12$. The transition flow characteristics are vey well portrayed. For $k n_{0}=0.5$ and 1.0, $\ell=1,2,4,8$ and 12, and $\alpha=0.1,0.5$ and 0.8 , the calculated value of the mass flux agrees to within $5 \%$ of the value of the mass flux obtained by using the plane Poiseuille formula with slip boundary conditions.

2. The discrete velocity ordinate method is used; the velocity field is represented by two speeds and eight discrete points in the angular space. The integration over the speeds of the velocity field is done by a Gaussian quadrature. The integration over the angular space is done by two methods; first by a simple trapezoidal integration, and then by a cotangent integration that accounts for the special discontinuous nature of the distribution function in the free molecule limit. The free molecule and transition flow solutions are obtained by both forms of angular integration. The range of parameters considered is the same as for the moment method. Compared to the solutions of 
Reynolds and Richey (1967), the free molecule solution by the discrete velocity ordinate method with trapezoidal:integration overestimates the mass flux by as much as $25 \%$ for $\ell=12$. However; the cotangent integration in view of its special construction represents the free molecule solution very well. For the transition flow calculations, the results by the two methods of angular integration vary less and less as $\mathrm{Kn}_{0}$ decreases:and $\ell$ increases. For the values of $\alpha$, and $\ell$ considered, the agreement with the moment method transition flow solutions is very good, having a maximum difference of $6 \%$ for $K n_{0}=5.0$. This difference decreases as $\mathrm{Kn}_{0}$ is decreased. The disadvantage of the method is the large amount of computer time required for the calculations.

3. For the range of parameters considered, the calculated value of the mass flux through the slot, $\dot{m}$, can be represented to within $5 \%$ by the following equation:

$$
\begin{aligned}
\frac{\dot{m}}{\rho_{0} a\left(2 R T_{0}\right)^{1 / 2}}= & \frac{1}{\ell}\left\{\frac{0.1331}{K_{0}}(1+\alpha) T_{1}+2\left(T_{1}-\frac{K n_{0}}{8} \log S_{1}\right)\right. \\
& \left.+\frac{K n_{0}}{8 \pi^{1 / 2}} \log S_{1} \log \left(\frac{\ell+\left(l^{2}+4\right)^{1 / 2}}{2}\right)\right\},
\end{aligned}
$$

where,

$$
\begin{aligned}
& T_{1}=\frac{(1-\alpha) \ell}{\ell+4.55-2.85 \exp \left\{0.21\left(\mathrm{Kn}_{0}-5\right)\right\}} \\
& S_{1}=\frac{2 K n_{0}+8(1+\alpha+T)}{2 K n_{0}+8(1+\alpha-T)}
\end{aligned}
$$

$R$ is the gas constant, ' $a$ '. the slot half width, , $\rho_{0}$ and $T_{0}$ the upstream density and temperature, respectively. 


\section{REFERENCES}

1. Anderson, D. G. (1967) "On the steady Krook kinetic equation: Part 2," J. of Plasma Physics 1, Pt. 2, 255-265.

2. Bhatnagar, P. L., Gross, E. P. and Krook, M. (1954), "A kinetic approach to collision processes in gases. I. Small amplitude processes in charged and neutral one component systems," Phys. Rev. 94, 511.

3. Bird, G. A. (1969) "Direct simulation Monte Carlo Method-current status and prospects," in Rarefied Gas Dynamics (ed. L. Trilling and H. Y. Wachman), Vol. I, Academic Press, New York, pp. 85-98.

4. Cercignani, C. and Daneri (1963) "Flow of a rarefied gas between two parallel plates," J. Appl. Phys. 34, 12, 3509-3513.

5. Cercignani, C. and Pagani, C. D. (1966) "Variational approach to boundary value problems in kinetic theory," Phys. of Fluids $\underline{9}, 1167$.

6. Fryer, G. M. (1966) "A theory of gas flow through capillary tubes," Proc. Roy. Soc. 293 A, 329-341.

7. Hamel, B. B. and Willis, D. R. (1966) "Kinetic theory of source flow expansion with application to the free jet," Phys. of Fluids $\underline{9}, 5,829$.

8. Hilby, D. and Pahl, W. (1952) "Correlation for plane Poiseuille transition flows," Z. Naturforsch. 7A, 542-549.

9. Huang, A. B. and Hartley, D. L. "Kinetic theory of the sharp leading edge problem in supersonic flow," Phys. of Fluids 12, 1, 96-108. 
10. Kennard, E: H. "(1938) Kinetic Theory of Gases, McGraw-Hi11 Book Co., Inc., New York.

11. Kopal, 2. (1961) Numerical Analys is, J. Wiley and Sons Inc, New York.

12. Lees, L. (1965) "A kinetic theory description of rarefied gas flows," J. Soc. Industrial Applied Math. 13, 278.

13. Liepmann, H. W., Narasimha, R. and Chahine, M. T. (1962) "Structure of a plane shock layer," Phys. of Fluids $\underline{5}$, $11,1313-1324$.

14. Liu, C. Y. (1968) "Plane Poiseuille flow of a rarefied gas," Phys. of Fluids 11, 3, 481-485.

15. Ortloff, C. R. (1968) "Restricted variational principle method for the free molecular mixing of parallel streams," J. Optimization and Application 2, 3, 187-198.

16. Pao, Y. P. and Willis, D. R. (1963) "A note on plane Poiseuille flow of a rarefied gas," AIAA Journal 1, 5, 1198.

17. Reynolds, T. W. and Richey, E. A. (1967) "Free molecule flow and surface diffusion through slots and tubes--A summary," NASA Tech. Rept. NASA TR R-255.

18. Rosen, P. (1954) "The solution of the Boltzmann equation for a shock wave using a restricted variational principle," J. Chem. Phys. 22, 6, 1045-1049.

19. Saranson, L. (1962) "On boundary value problems for hyperbolic equations," Comm. Pure and Applied Math. 15, 373-395.

20. Sherman, F. S. (1963) "A survey of experimental results and methods for the transition regime of rarefied gas dynamics," 
in Rarefied Gas Dynamics (ed. J. A. Laurmann), VoT. II, Academic Press, pp. 228-260. 


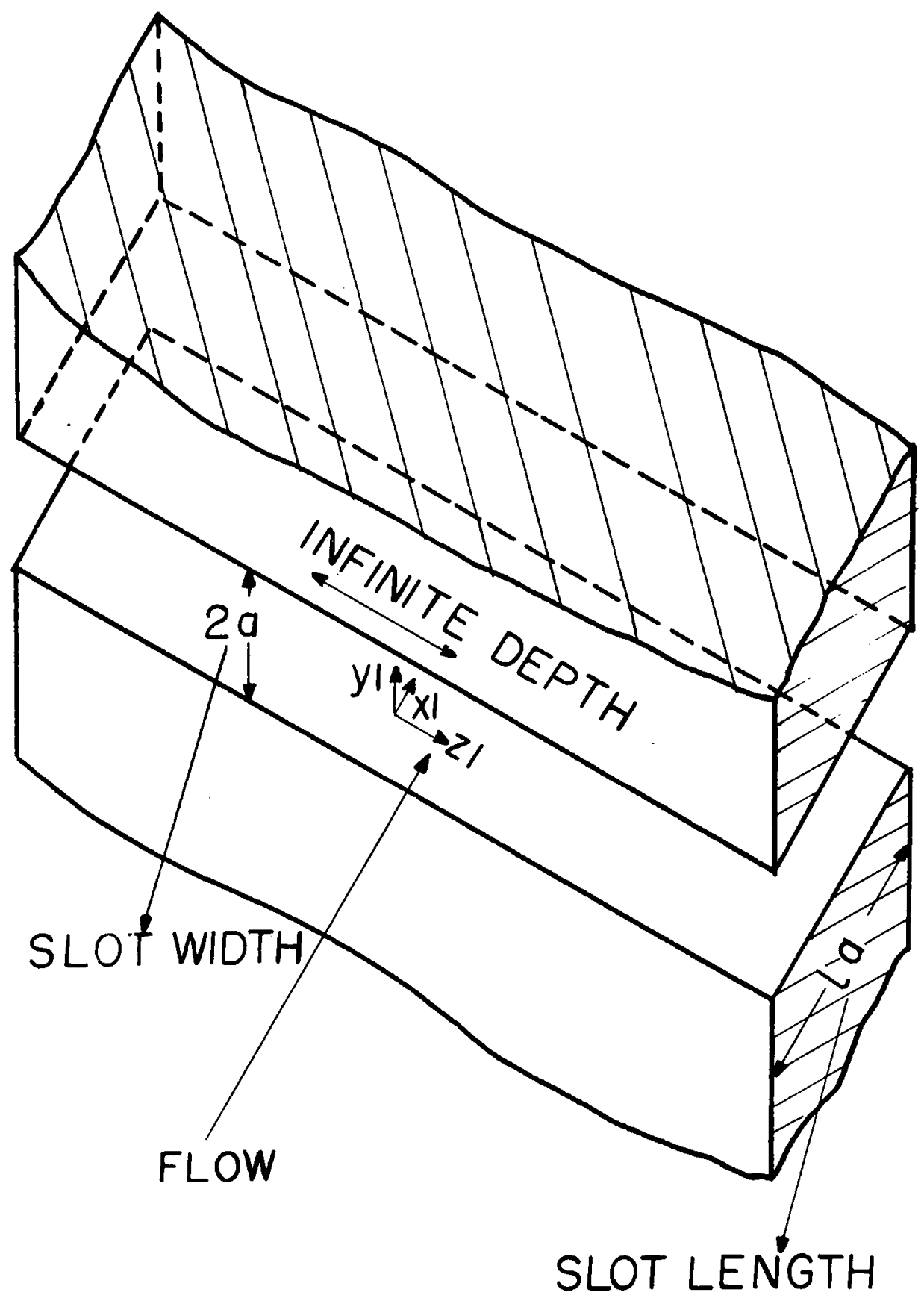

FIG.Ia SLOT GEOMETRY 


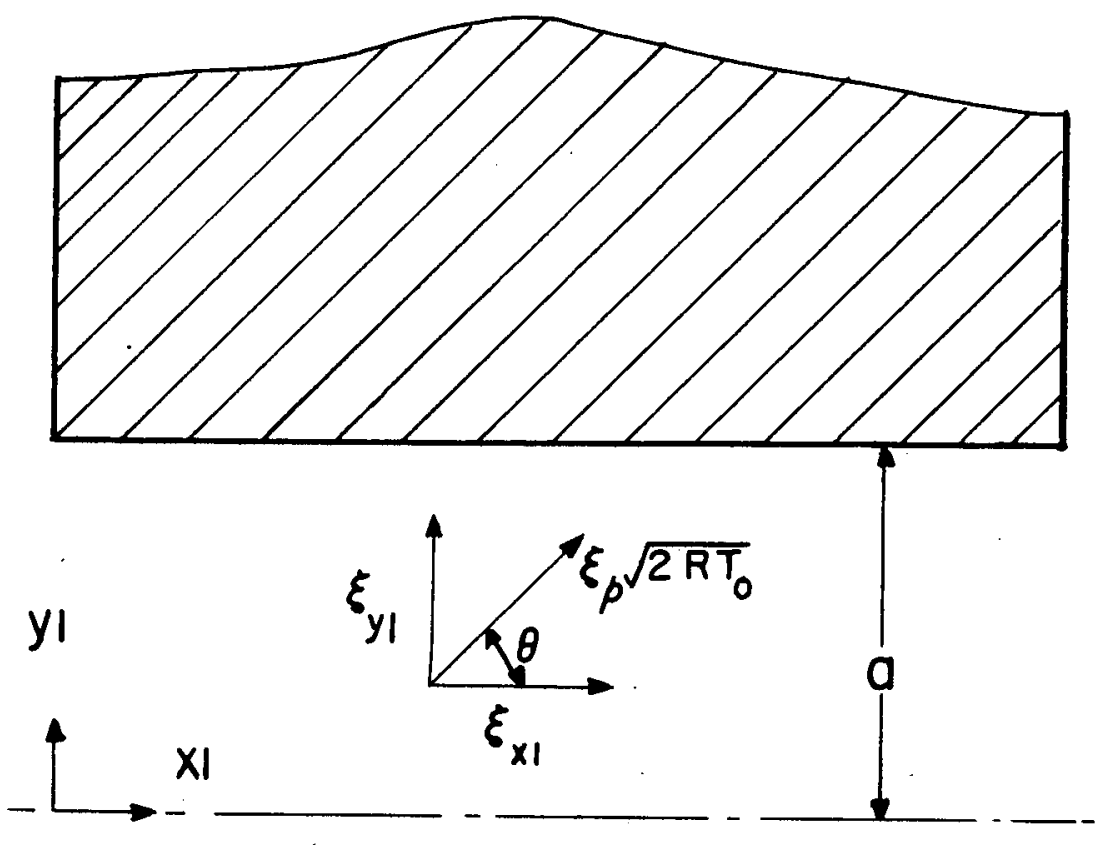

$\operatorname{ro}^{0}$

$\underbrace{0}_{\dot{0}}$

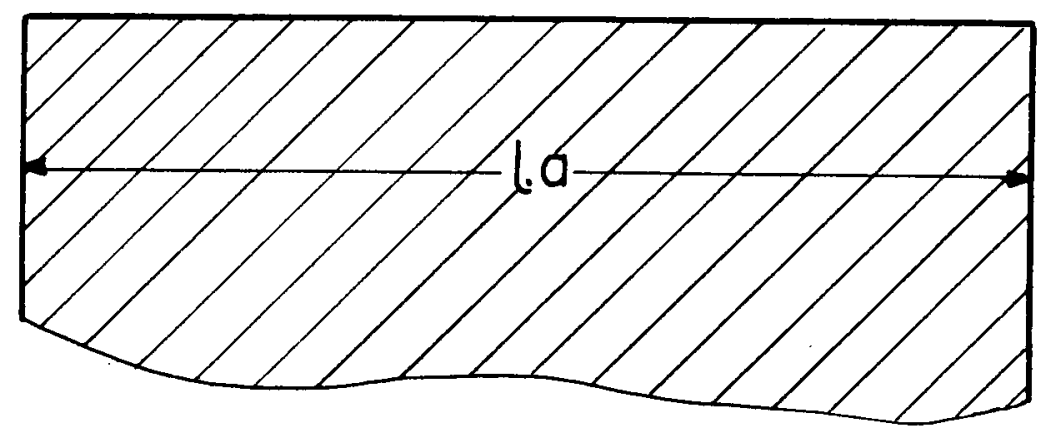

FIG. Ib SLOT GEOMETRY 


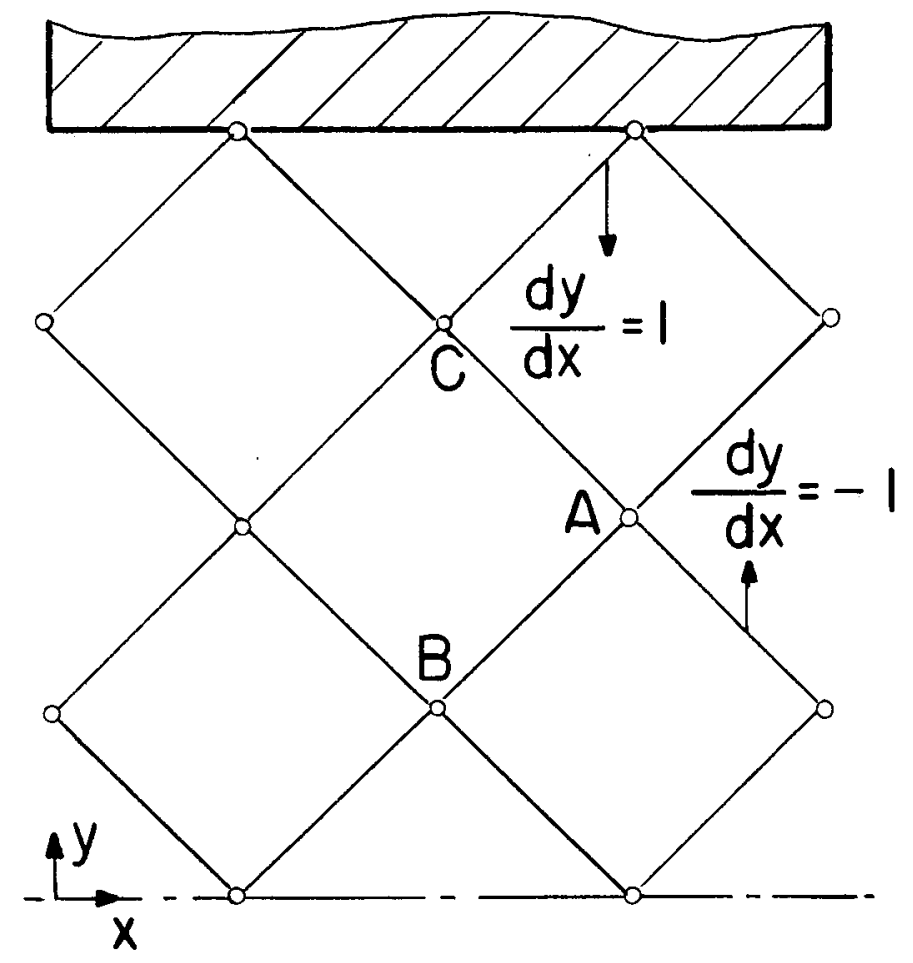

FIG.2 GRID POINTS FOR THE MOMENT METHOD

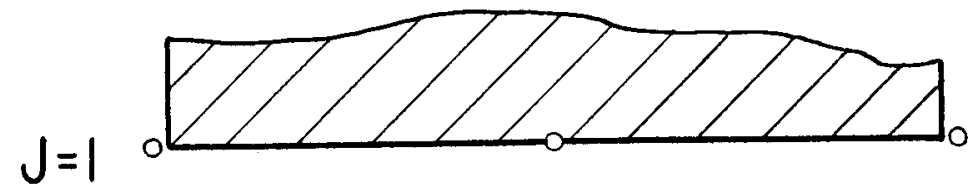

$J=2$ 。

0

$\circ$

$J=N N_{\circ}^{l=1}$
GIG 3 ORID POINTS FOR THE DISCRETE
ORDINATE METHOD 


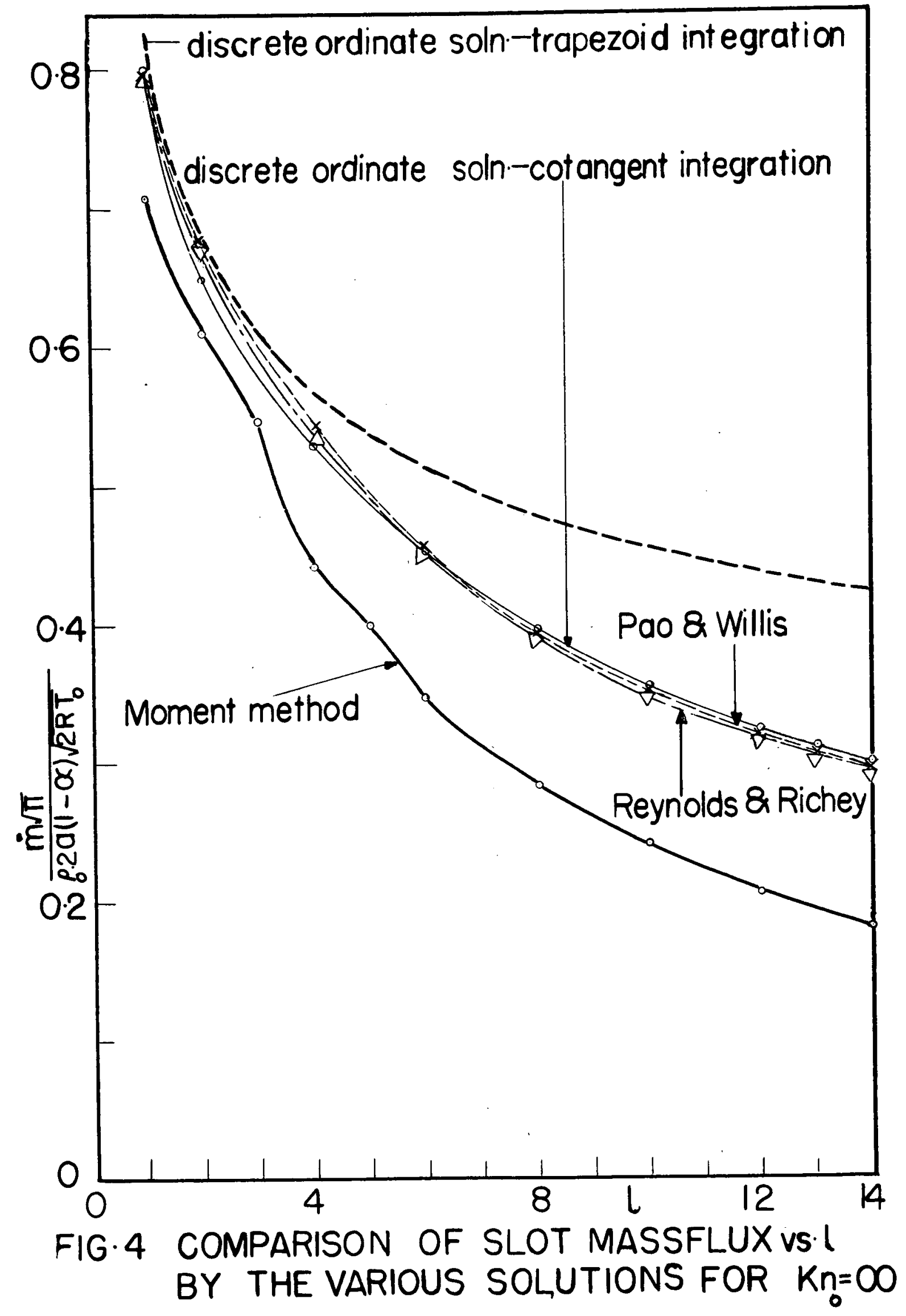




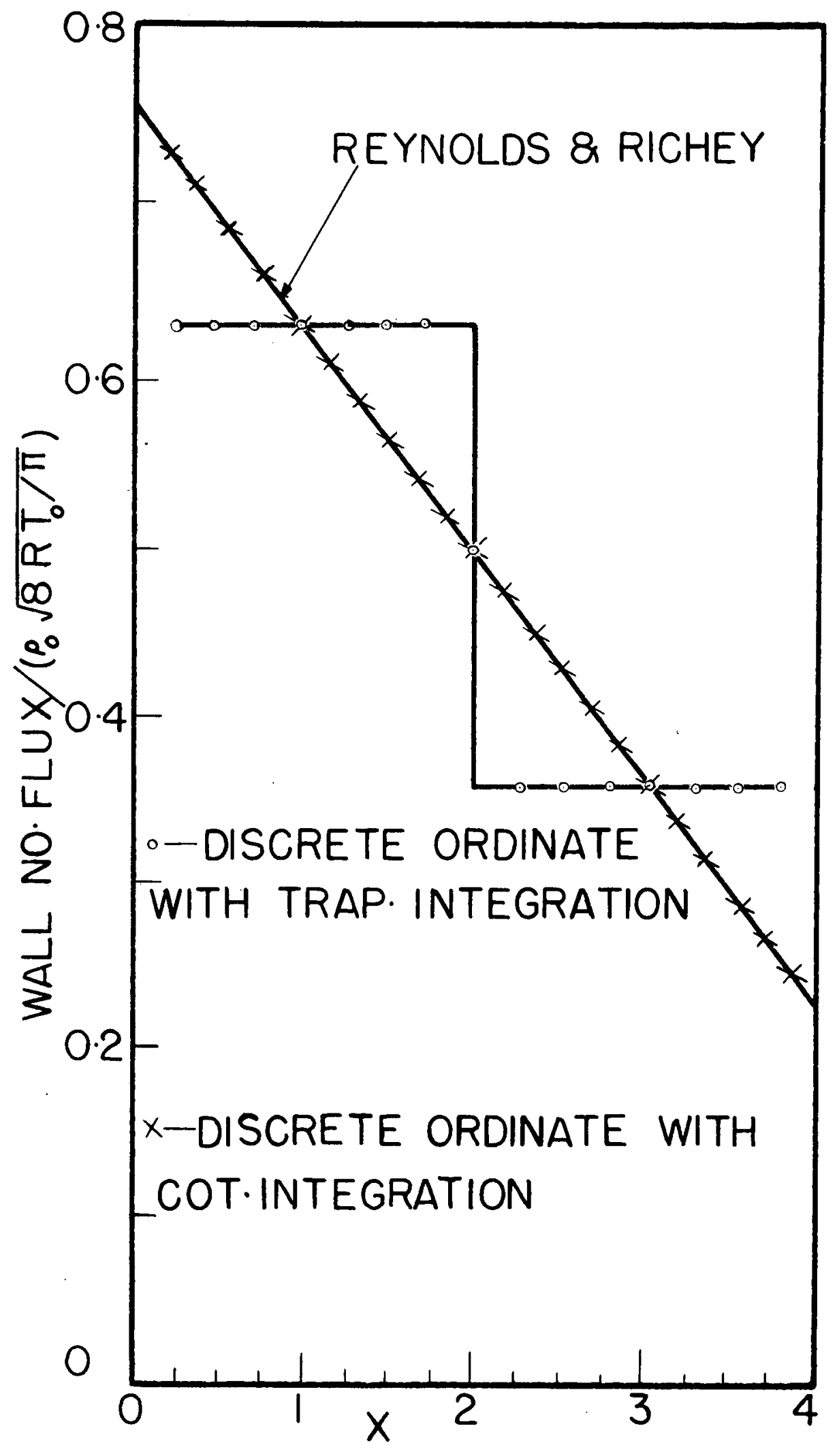

FIG 5 FREE MOLECULE WALL FLUX vs $X, l=4, \propto=0$ 


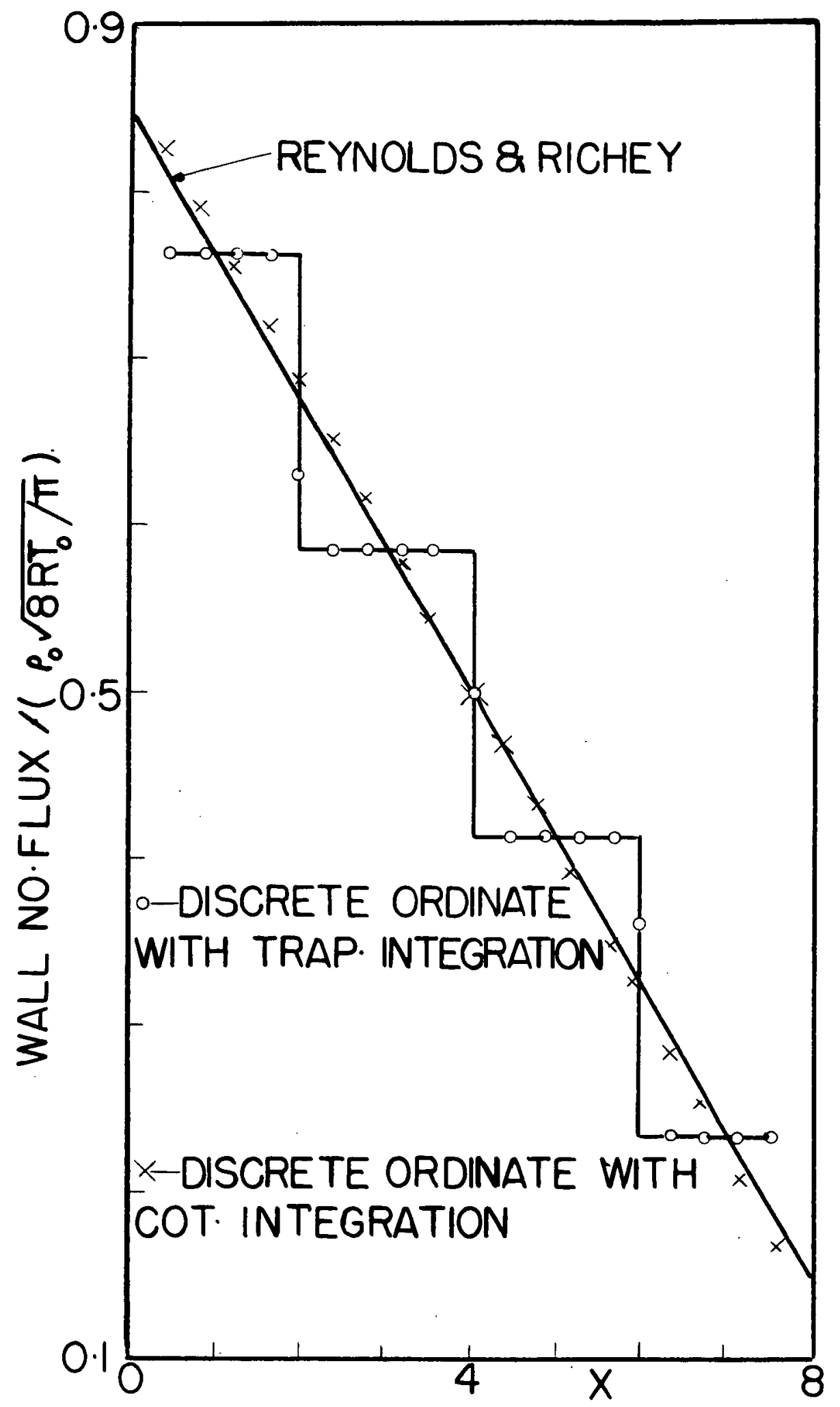

FIG. 6 FREE MOLECULE WALL NO. FLUX vs.X, $\mathrm{l}=8, \propto=0$. 


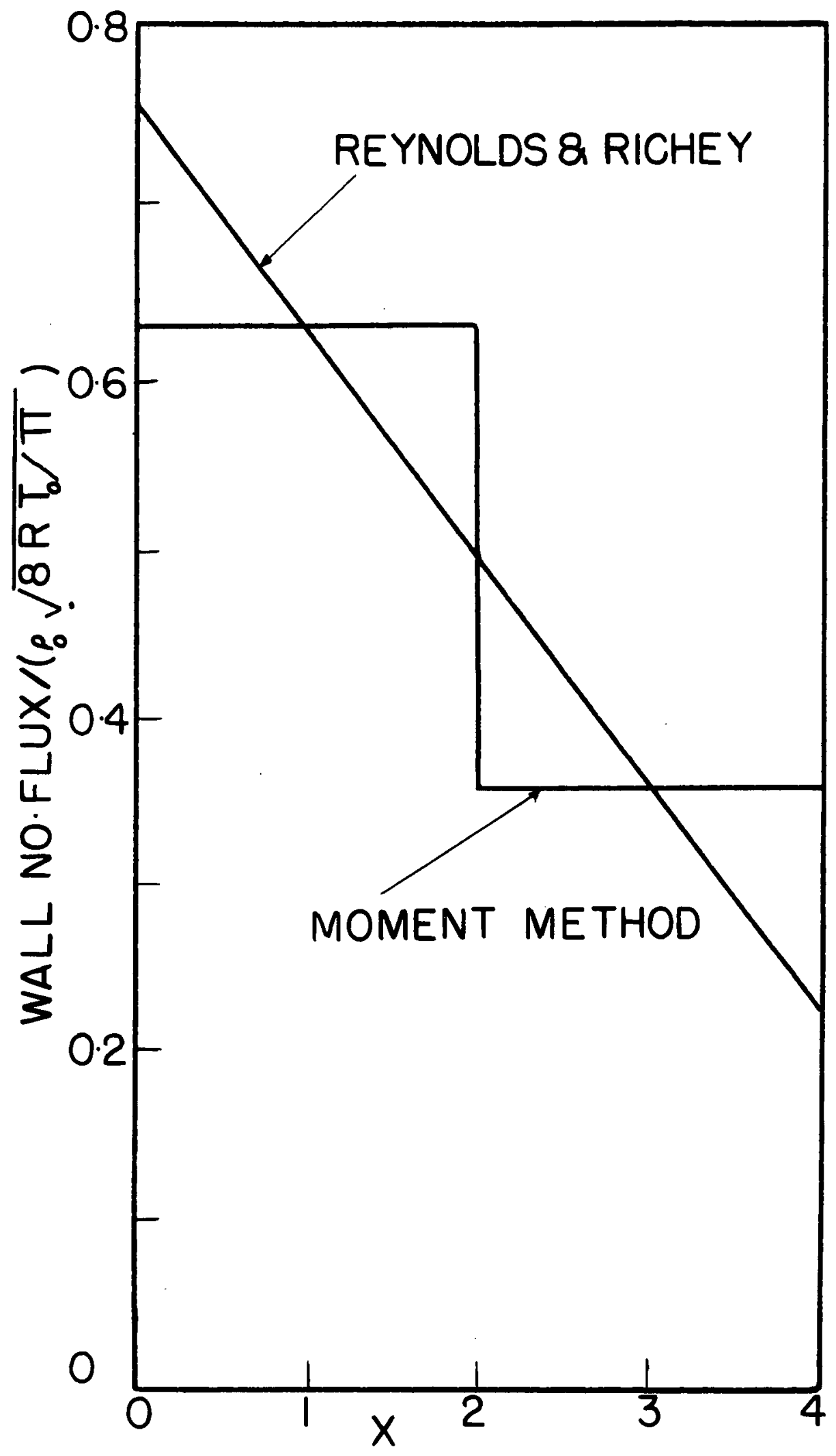

FIG. 7 FREE MOLECULE WALL FLUX vs $X, L=4, \alpha=0$ 


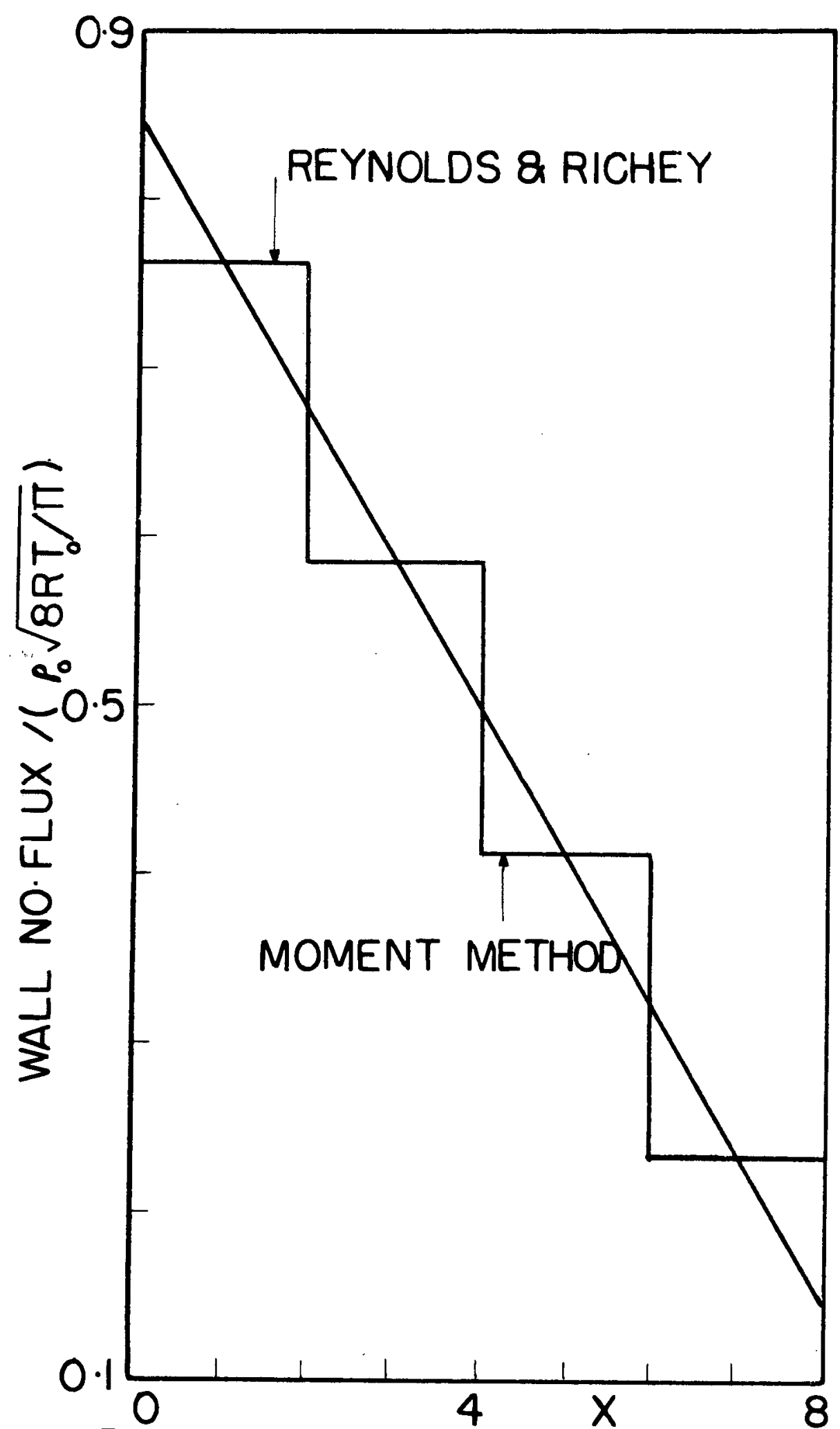

FIG. 8 FREE MOLECULE WALL NO. FLUX vs:X, $L=8, \propto=0$. 


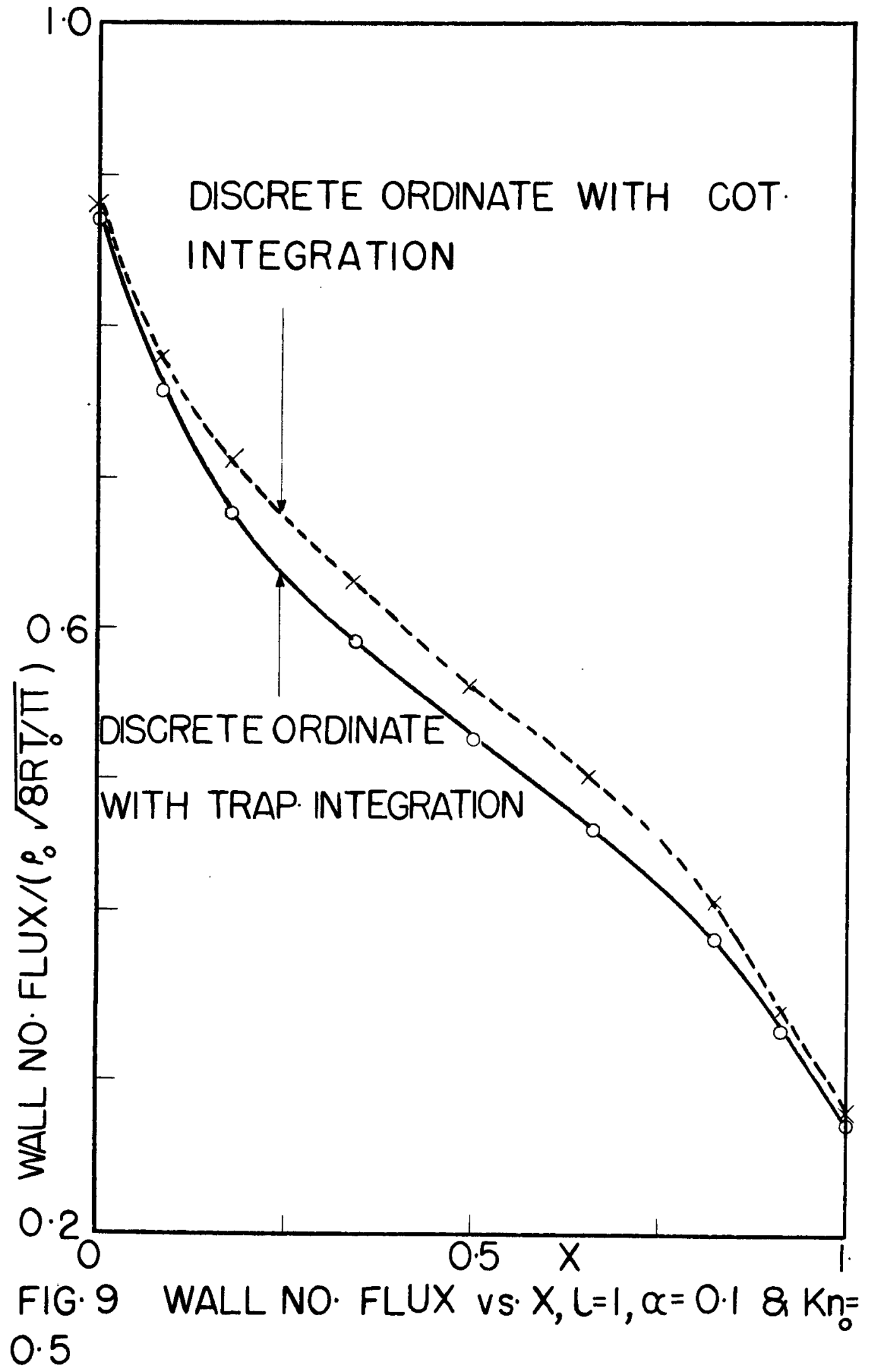




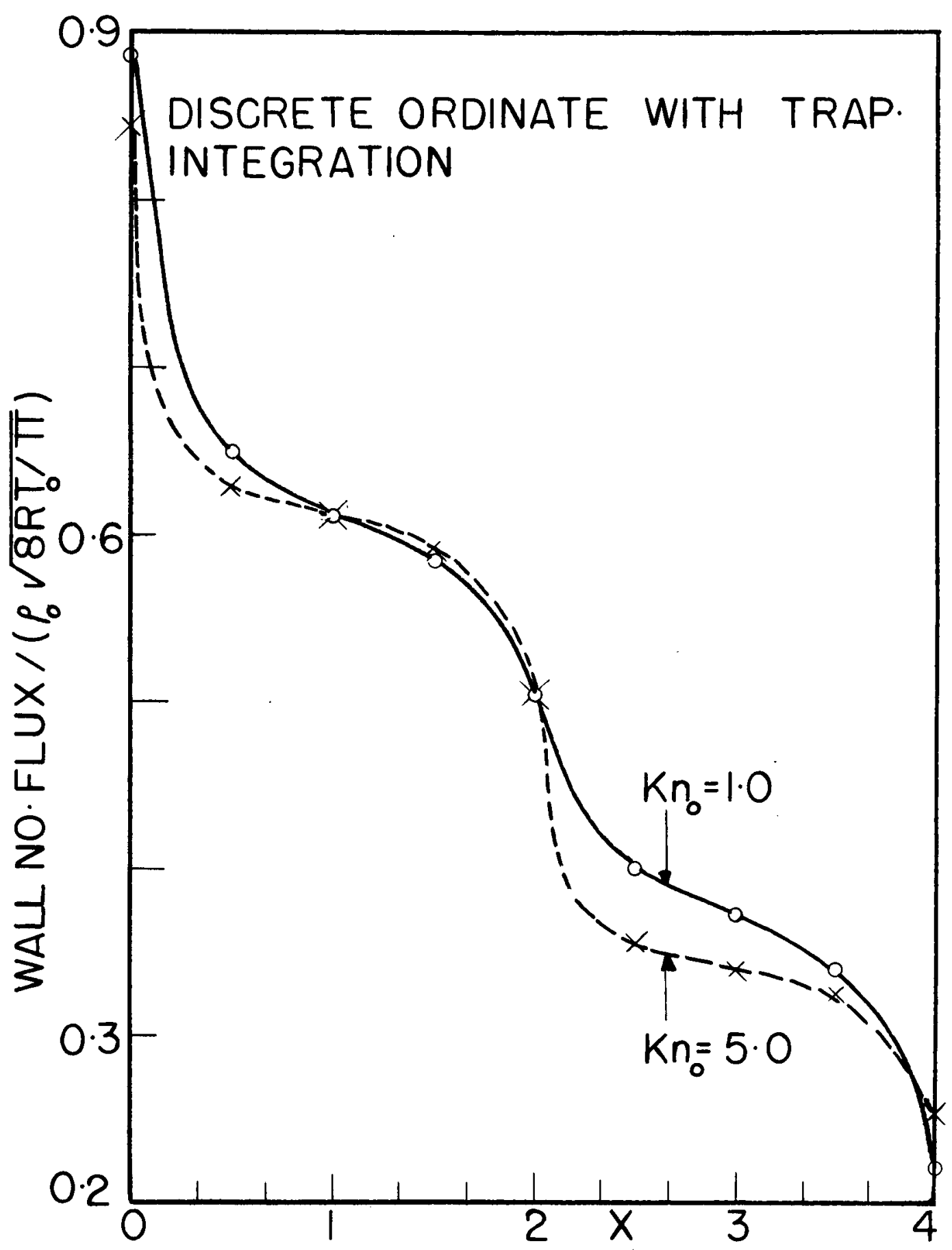

FIG. 10 WALL NO. FLUX vs. $X, L=4, \alpha=0 \cdot 1, K n_{0}=$ $1.0 \quad \& 5.0$ 


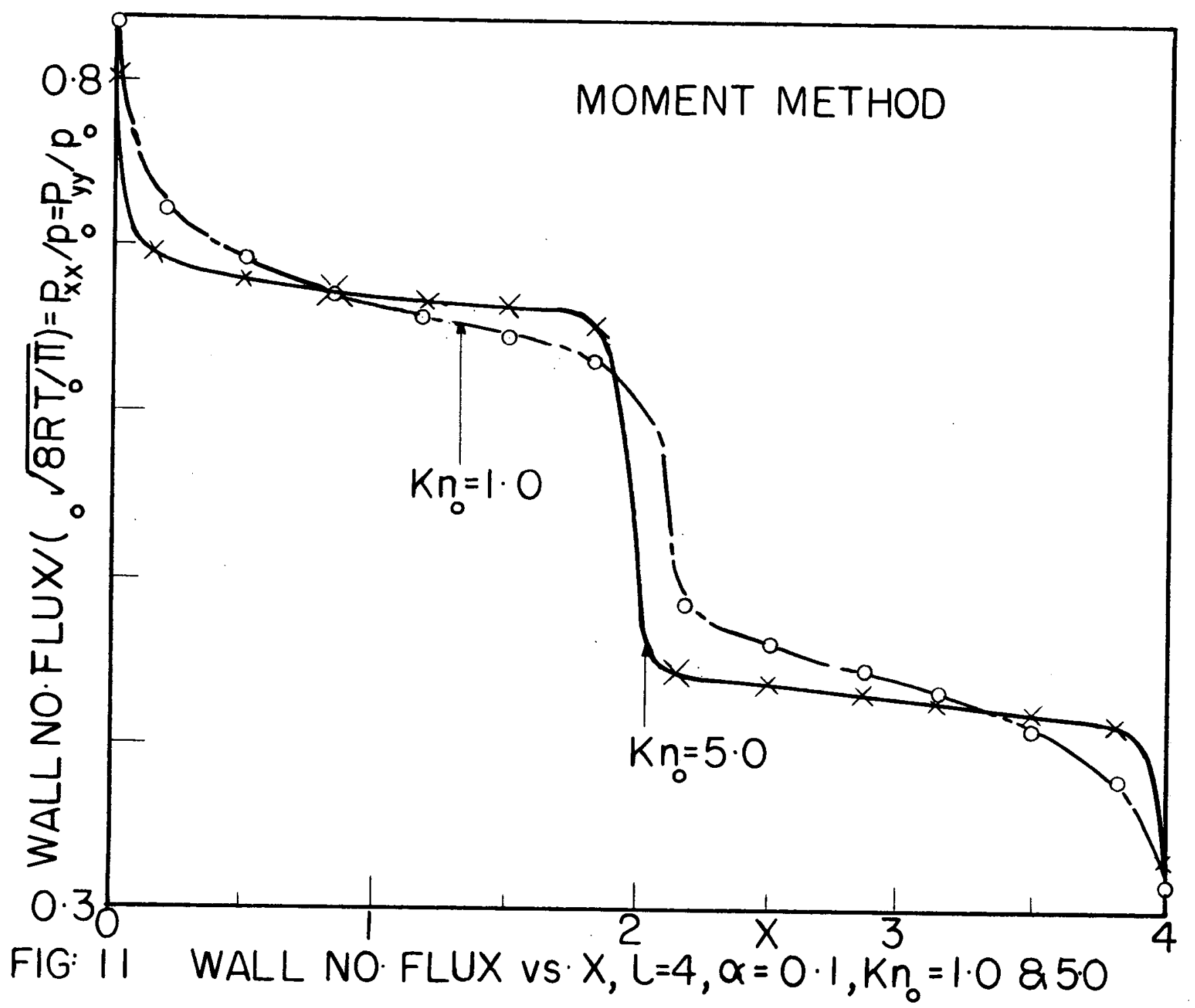




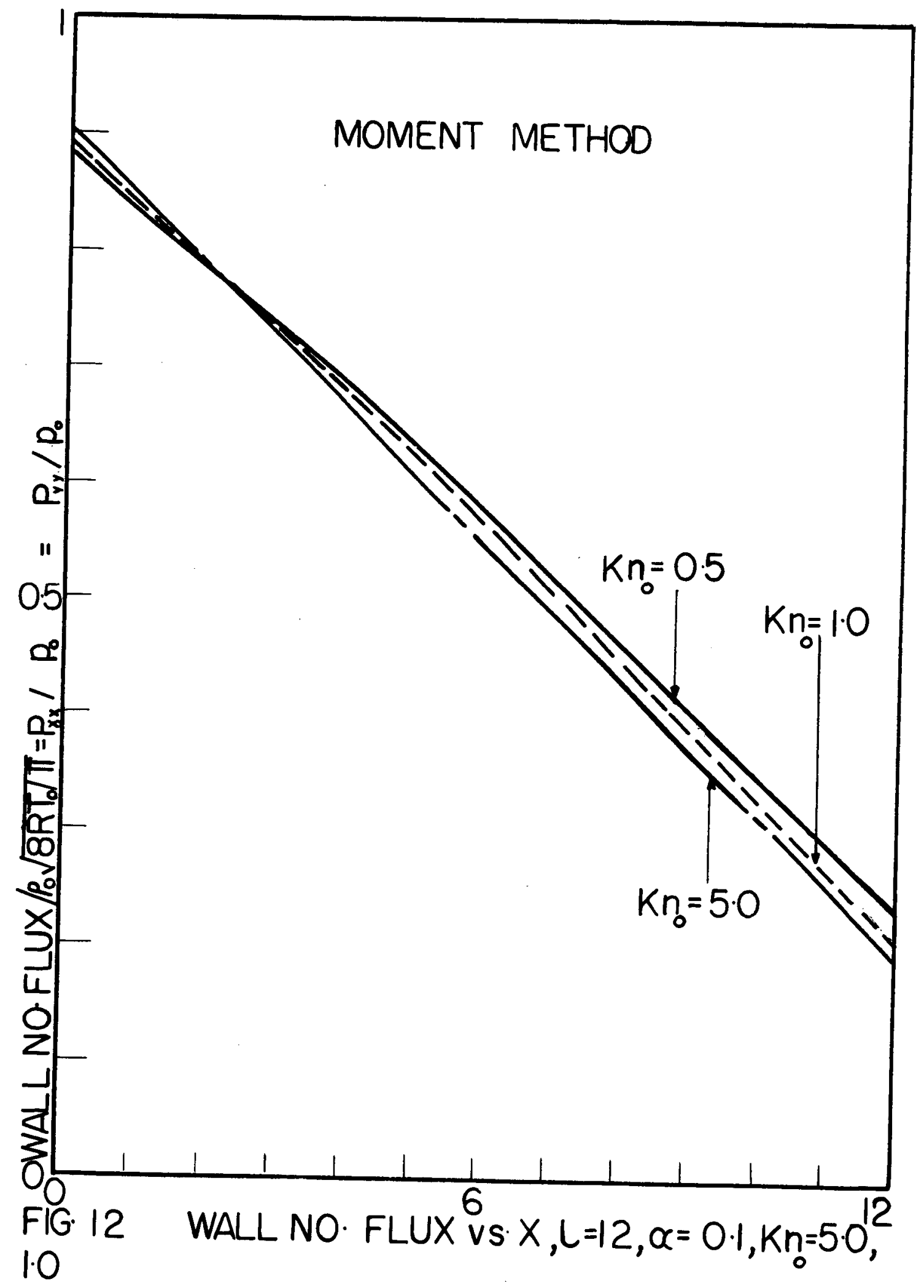




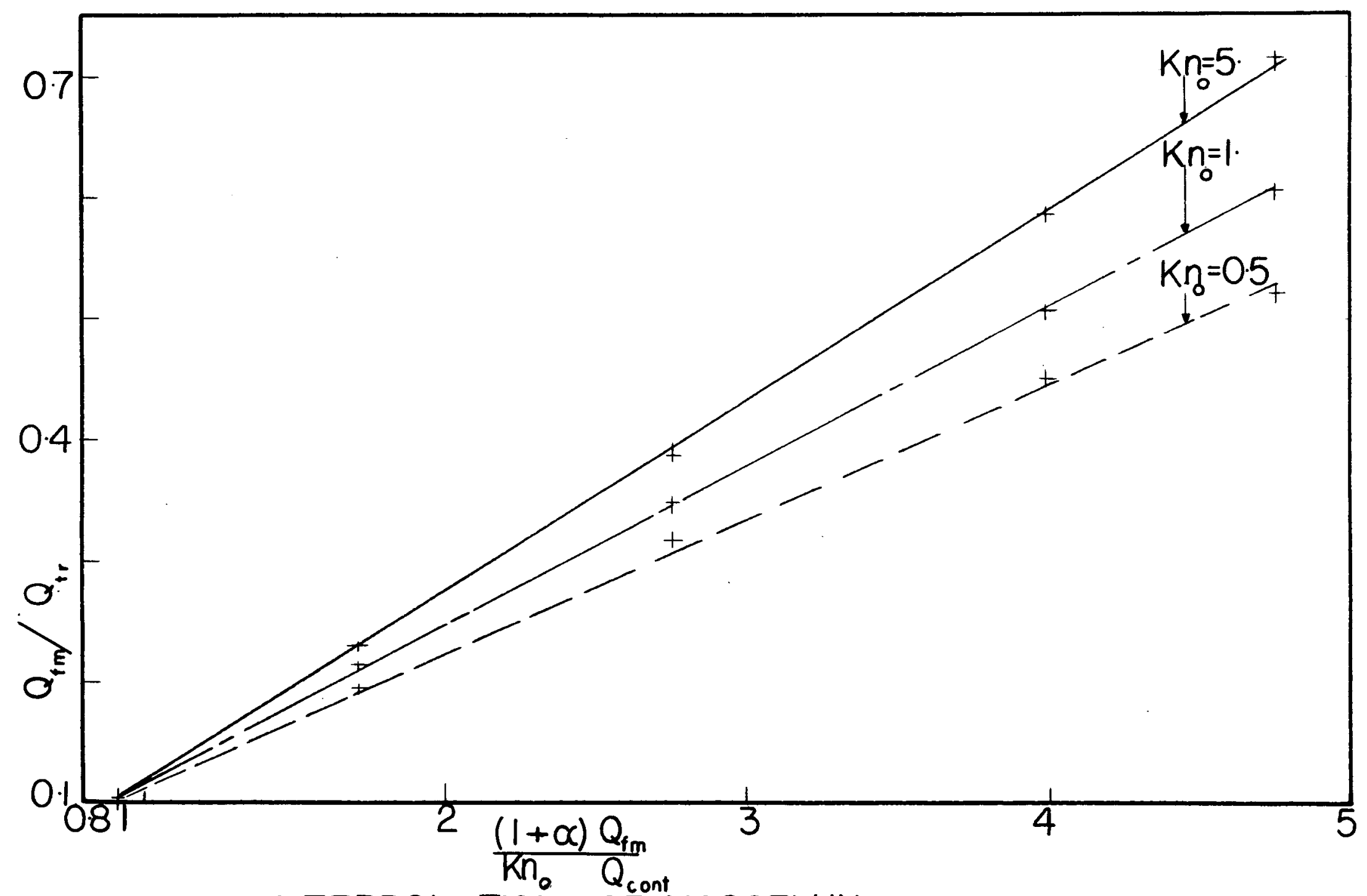

FIG. 13 INTERPOLATION OF MASSFLUX 


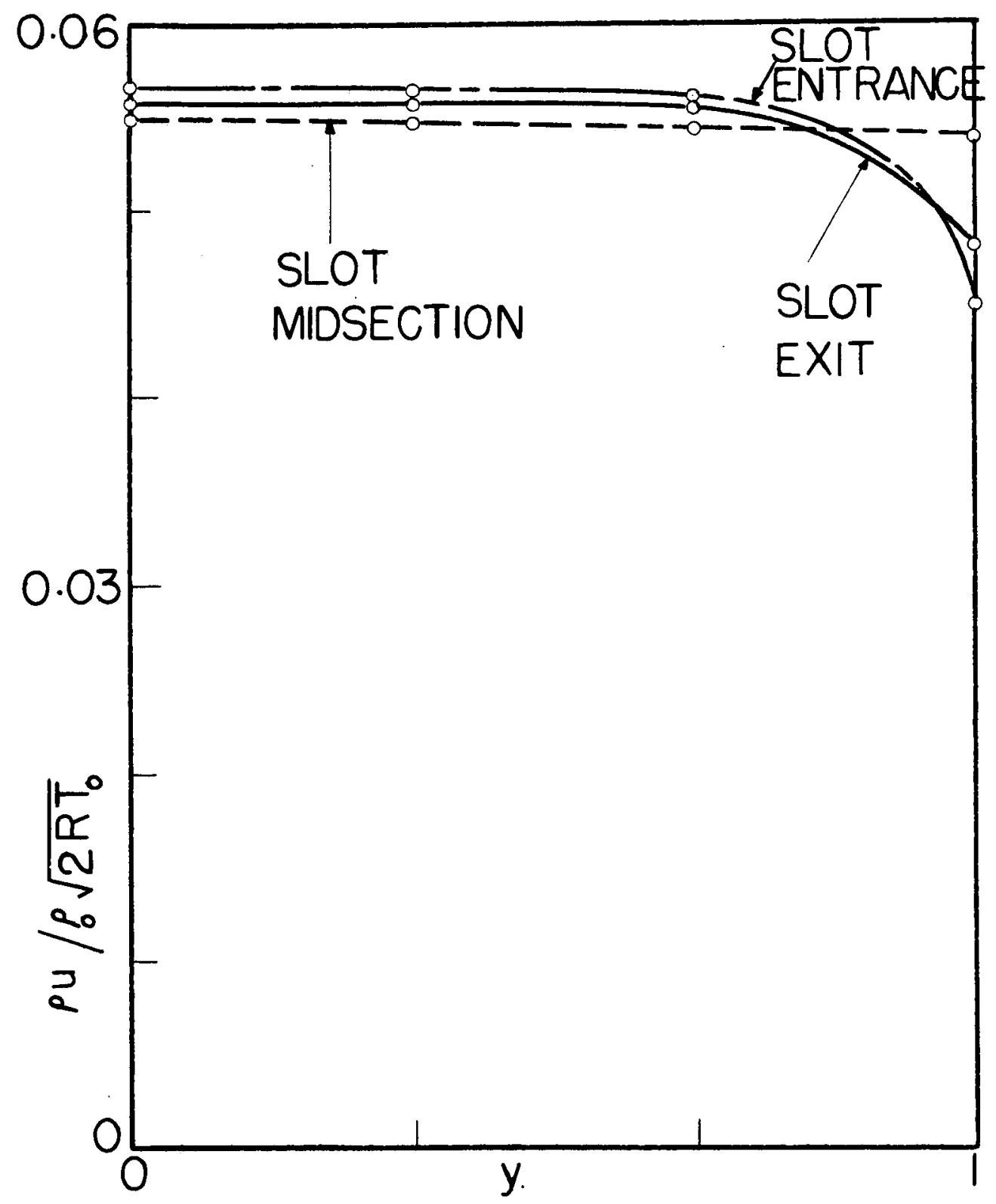

FIG. $14 \quad \rho u$ vs. $y, L=12, \alpha=0.1 \& K K_{0}=5 \cdot 0$ 


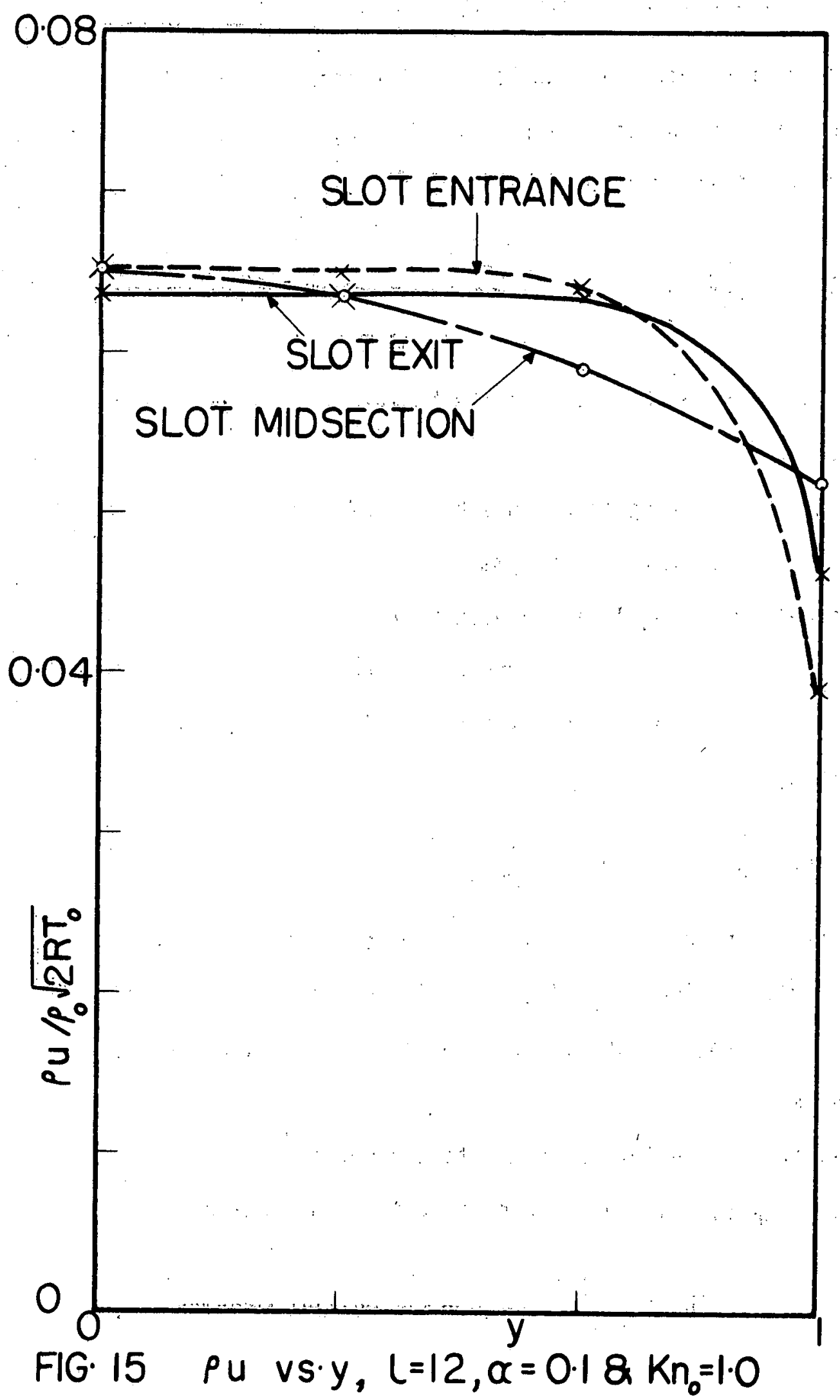




\section{APPENDIX A}

\section{EXISTENCE AND UNIQUENESS OF MOMENT SOLUTIONS}

The source material used here is Saranson's work (1962).

Let $(n)$ be a vector function of $\left(x_{1}, x_{2}, \ldots, x_{N}\right)$. In a region $R$, let it satisfy the boundary differential equation,

$$
\sum_{i=1}^{N} A_{i j} \frac{\partial n_{j}}{\partial x_{i}}+C_{j} n_{j}=0
$$

On the boundary $S$ of $R$, let the boundary condition $(H)(n)=(g)$ be specified.

Let the following conditions be satisfied:

1) The matrices $\left(A_{i j}\right)$ are symmetric.

2) The matrices $\left(A_{1 j}\right)$ are nonsingular.

3) $\left(A_{1 j}\right)^{-1}$ and $\left(C_{j}\right)$ are bounded matrices.

4) The roots of the characteristic equations are real.

5) Define,

$$
(k)=\frac{1}{2}\left((c)+(C)^{\prime}-\sum_{i=1}^{N} \frac{\partial A_{i j}}{\partial x_{i}}\right)
$$

where the prime sign ' is used to denote the transpose of the matrix. Then, $\frac{1}{2}\left((k)+(k)^{\prime}\right)>0$. (If this condition is satisfied, the system is said to be a positive symmetric system.)

6) Call the unit exterior normal to the surface $S$ as $(\theta)=\left(\theta_{0}, \theta_{1}, \ldots, \theta_{r}\right)$. Along $S$, define the matrix

$$
(B)=\frac{1}{2} \sum_{i=0}^{r} \theta_{i} A_{i j}
$$


and write, $(B)=\left(B_{+}\right)+\left(B_{-}\right)$and $(m)=\left(B_{+}\right)-\left(B_{-}\right)$. Then (m) has a nonnegative symmetric part.

7) If the above six conditions are satisfied and $(N)=(m)-(B)$ with $(g)$ in the range of $(N)$, then $(N)(n)=(g)$ is said to be an admissible boundary condition. That is, for the given differential equation and boundary condition, a unique solution exists.

Considering our problem, define

$$
Q=\frac{\sqrt{\pi}}{K n_{0}\left(T / T_{0}\right)^{\omega-T}}
$$

and

$$
(M)=\left[\begin{array}{l}
M_{00} \\
M_{01} \\
M_{10} \\
M_{11}
\end{array}\right]
$$

Neglect $M_{10} \cdot M_{11}$ compared to $M_{11}$ in the last equation of the set of equations (3.11). Our equations and boundary conditions then have the form,

$$
\begin{aligned}
& \left\{\left(\begin{array}{cccc}
\frac{1}{2} & 0 & 0 & 0 \\
0 & 0 & 1 & 0 \\
0 & 1 & 0 & 0 \\
0 & 0 & 0 & 2
\end{array}\right) \frac{\partial}{\partial x}+\left(\begin{array}{llll}
0 & 0 & 0 & 1 \\
0 & 1 & 0 & 0 \\
0 & 0 & 1 & 0 \\
1 & 0 & 0 & 0
\end{array}\right) \frac{\partial}{\partial y}\right. \\
& +\left(\begin{array}{llll}
0 & 0 & 0 & 0 \\
0 & 0 & 0 & 0 \\
0 & 0 & 0 & 0 \\
0 & 0 & 0 & 0
\end{array}\right)
\end{aligned}
$$


with,

$$
\begin{aligned}
& \left(\begin{array}{cccc}
1 & 0 & \sqrt{\pi} & 0 \\
0 & 0 & 0 & 0 \\
0 & 1 & 0 & \sqrt{\pi} \\
0 & 0 & 0 & 0
\end{array}\right) \quad(M)=\left(\begin{array}{l}
1 \\
0 \\
0 \\
0
\end{array}\right) \text { at } x=0, \\
& \left(\begin{array}{cccc}
1 & 0 & -\sqrt{\pi} & 0 \\
0 & 0 & 0 & 0 \\
0 & 1 & 0 & -\sqrt{\pi} \\
0 & 0 & 0 & 0
\end{array}\right) \quad(M)=\left(\begin{array}{l}
\alpha \\
0 \\
0 \\
0
\end{array}\right) \text { at } x=\ell
\end{aligned}
$$

and

$$
\left(\begin{array}{cccc}
0 & 0 & 0 & 0 \\
0 & 1 & 0 & 0 \\
-\sqrt{\pi} & 0 & 1 & 0 \\
0 & 0 & 0 & 0
\end{array}\right) \quad(M)=\left(\begin{array}{l}
0 \\
0 \\
0 \\
0
\end{array}\right) \text { at } y=0 \text { and } y=1
$$

Replace (M) by

$$
(G)=\left(\begin{array}{l}
G_{00} \\
G_{01} \\
G_{10} \\
G_{11}
\end{array}\right)
$$

as the unknowns, where

$$
(M)=\left(\begin{array}{llll}
p & m & \ell & n \\
m & r & q & \ell \\
\ell & q & r & m \\
n & \ell & m & p
\end{array}\right)
$$

and $\ell, m, n, p, q$ and $r$ are arbitrary functions whose choice is at our discretion.

Substituting for (M) in Eq. (A.1), we have 


$$
\left[\left(A_{1}\right) \frac{\partial}{\partial x}+\left(A_{2}\right) \frac{\partial}{\partial y}+\left(C_{1}\right)\right](G)=0,
$$

where

and

$$
\begin{aligned}
& \left(A_{1}\right)=\left(\begin{array}{llll}
p & m & \ell & n \\
m & r & q & \ell \\
\ell & q & r & n \\
n & \ell & m & p
\end{array}\right) \\
& \left(A_{2}\right)=\left(\begin{array}{llll}
n & l & m & p \\
l & q & r & m \\
m & r & q & \ell \\
p & m & l & n
\end{array}\right)
\end{aligned}
$$

$$
\left(C_{1}\right)=\frac{\partial}{\partial x}\left(A_{1}\right)+\frac{\partial}{\partial y}\left(A_{2}\right)+Q \quad\left(\begin{array}{llll}
0 & 0 & 0 & 0 \\
0 & 0 & 0 & 0 \\
0 & 0 & 0 & 0 \\
n & \ell & m & p
\end{array}\right)
$$

Condition 1$)$ is satisfied since $\left(A_{1}\right)$ and $\left(A_{2}\right)$ are symmetric matrices. By an appropriate choice of $\ell, m, n, p, q$ and $r$ we can make $\left(A_{1}\right)$ nonsingular and $\left(A_{1}\right)^{-1}$ and $\left(C_{1}\right)$ bounded. Conditions 2) and 3) are thus satisfied.

Calculation of the roots of the characteristic equation yields values of 1 and -1 --both of which are real. Thus condition 4$)$ is satisfied. Further, we have $\frac{1}{2}\left((k)+(k)^{\prime}\right)=$ $\frac{1}{4}\left(\left(c_{1}\right)+\left(c_{1}\right)^{\prime}\right)$. Thus if we choose $\ell, m, n, p, q$ and $r$ such that $\ell_{x}, \ell_{y}, m_{x}, m_{y}, \ldots, r_{x}, r_{y}$ are all positive, then $\frac{1}{2}\left((k)+(k)^{\prime}\right)=\frac{1}{4}\left(\left(c_{1}\right)+\left(c_{1}\right)^{\prime}\right)$ is greater than zero. Thus condition 5) is satisfied, i.e., the system is symmetric positive.

Finally, choose $\ell, m, n, p, q$ and $r$ such that they are all positive in our domain, and also such that $p>2 \sqrt{\pi} m>4 \pi r$ and 
$n>2 \sqrt{\pi} \ell>4 \pi q$. Then conditions 6) and 7) are immediately satisfied.

A choice of $\ell, m, n, p, q$ and $r$ can easily be established such that the above conditions are satisfied. Thus a unique solution exists for $(G)$. But since $(M)$ is a simple algebraic combination of $(G)$, hence a unique solution exists for $(M)$ as we11. 


\section{APPENDIX B}

FREE MOLECULE FLOW RESULTS FOR THE MOMENT METHOD

Let us first consider the case where $l$ is an even integer, equal to $2 n$, where $n$ is an integer. Let us define

$$
D=\frac{(1-\alpha)}{4(1+l / \pi)}
$$

Then,

$$
\begin{array}{ll}
H(S)=D / \sqrt{\pi} & \text { for }-1 \leq S \leq 2 n+1, \\
G(S)=\frac{1}{2}-D & \text { for }-1 \leq S \leq 1, \\
G(S)=\frac{\alpha}{2}-D \quad & \text { for } \ell-1 \leq S \leq 2 n+1, \\
G(S)=\frac{1}{2}-\left\{1+\frac{4}{\pi}(m+1)\right\} D
\end{array}
$$

for

$$
1+2 m<S \leq 1+2(m+1) \text { with } m=0,1, \ldots,(n-3),
$$

while

$$
G(S)=\frac{1}{2}-\left\{1+\frac{4}{\pi}(n-1)\right\} D
$$

for

$$
2 n-3<S<2 n-1
$$

Secondly, let us consider the case where $\ell$ is an odd integer, equal to $(2 n+1)$, where $n$ is an integer.

Let us first define the following quantities:

$$
E=\frac{(1-\alpha)}{4\left(1+\frac{2 n}{\pi}\right)}
$$


and

$$
B=\frac{1-\alpha}{2 \pi(2 n+3)+4\left(2 n^{2}+4 n+1\right)}
$$

Then,

$$
\begin{array}{ll}
H(S)=E / \sqrt{\pi} & \text { for } S=-1,0,1, \ldots,(2 n+2), \\
H(S)=(n+1) \sqrt{\pi} B & \text { for } 2 m>S>2 m-1, \\
H(S)=(n+2) \sqrt{\pi} B & \text { with } m=0,1,2, \ldots, n+1, \\
& \text { for } 2 m+1>S>2 m \\
& \text { with } m=0,1,2, \ldots, n-1,
\end{array}
$$

while,

$$
\begin{array}{ll}
G(S)=\frac{1}{2}-E & \text { for } S=-1,0,1 \\
G(S)=\frac{1}{2}-E-\frac{4 m}{\pi} E & \text { for } S=2 m \text { or } 2 m+1 \\
\text { with } m=1,2, \ldots, n, \\
G(S)=\frac{\alpha}{2}+E & \text { for } S=2 n+2 \\
G(S)=\frac{1}{2}-\frac{\{(2 n+3) \pi-2\}}{2} B-4 m(n+1) B & \text { for } 2 m>S>2 m-1 \\
& \text { with } m=0,1,2, \ldots, n+1,
\end{array}
$$

and

$$
\begin{aligned}
& G(S)=\frac{1}{2}-\frac{1}{2}\{(2 n+3) \pi+2\} B-4 m(n+2) B \\
& \qquad \begin{aligned}
& \text { for } 2 m+1>S>2 m \\
& \text { with } m=0,1, \ldots,(n-1)
\end{aligned}
\end{aligned}
$$

As noted in the text, solutions for $G$ and $H$ can be obtained for any rational value of $\ell$. Except for integer values of $\ell$, the expressions for $G$ and $H$ are very complicated. 


\section{APPENDIX C}

\section{ITERATION SCHEME FOR INITIAL VALUES}

Using either the moment or discrete ordinate method we have a problem where, in the discrete formulation, we have to determine a simultaneous set of values $q_{1}, q_{2}, \ldots, q_{m}$ such that

$$
\left(p_{1}, p_{2}, \ldots, p_{m}\right)=M\left[\left(q_{1}, q_{2}, \ldots, q_{m}\right)\right]
$$

or

$$
p=M(q)
$$

The nonlinear operator $M$ represents the marching procedure described in the text, $\mathrm{p}$ represents the given boundary values at $x=\ell$ (and the wall for the discrete ordinate method) and $q$ the unknown boundary values at $x=0$ (and the wall for the discrete ordinate method).

To solve (C.1) we use an iterative method based on linearizing Eq. (C.1). Let $q^{n}$ be an approximation to the solution with

$$
\underline{p}^{n}=M\left(q^{n}\right)
$$

If $q^{n}$ is close to the true solution $q$ it seems reasonable to assume that $\mathrm{p}-\mathrm{p}^{\mathrm{n}}$ is, to good approximation, linearly dependent on $\left(q-q^{n}\right)$. We therefore calculate $q^{n+1}$ using

$$
\left(p_{i}^{n+1}-p_{i}^{n}\right)=\sum_{j=1}^{m} A_{i j}{ }^{n}\left(q_{j}^{n+1}-q_{j}^{n}\right)
$$

The elements of the matrix $A_{i j}{ }^{n}$ are given for $i=1, \ldots, m$ by 


$$
A_{i j}{ }^{n}=\frac{\Delta p_{j}^{n}}{\Delta q_{j}}
$$

where $\Delta p_{i}{ }^{n}$ is the change produced in $p_{i}$ by varying $q_{j}^{n}$ to $q_{j}{ }^{n}+\Delta q_{j}$ while holding all other members of $q^{n}$ fixed. To determine all the members of $A_{i j}{ }^{n}$ we now vary $j$ from 1 to $m$. We have to "march" through the slot $m$ times to determine the matrix $A_{i j}{ }^{n}$.

The solution of Eq. (C.3) to obtain $q_{j}{ }^{n+1}$, for $j=1$ to $\mathrm{m}$, was done using the University of California Computer Center Library Subroutine LINEQF. An optimum value of $\Delta q_{j}(j=1$ to $m)$ exists for smooth and quick iteration to the initial values. All the $\Delta q_{j}$ 's $(j=1$ to $m)$ were set equal. The final choice of the single value, $\varepsilon$, was found to depend very critically upon $a$ and $K \mathrm{n}_{0}$. ranging from $10^{-4}$ to $10^{-7}$. A smaller value was required if $\alpha$ was increased and/or $K n_{0}$ was decreased. 


\section{APPENDIX D}

GAUSSIAN QUADRATURE FORMULAS FOR $\cdot \int_{0}^{\infty} d c \cdot c e^{-c^{2}} h_{1}(c)$

A general reference for the material here is Kopal (1961). We consider quadrature formulas of the form

$\int_{a}^{b} w_{1}(c) h_{1}(c) d c \approx \sum_{K=1}^{n} H_{K}^{(n)} h_{j}\left(c_{K}^{(n)}\right)$,

where $H_{K}(n)$ and $C_{K}{ }^{(n)}$ are the Gaussian quadrature weights and roots, respectively, in the interval $a<c<b$.

Define an inner product by

$$
\left(h_{1}, g_{1}\right)=\int_{a}^{b} w_{1}(c) h_{1}(c) g_{1}(c) d c
$$

There exists a set of polynomials

$$
p_{n}(c)=A_{n} c^{n}+B_{n} c^{n-1}+\ldots\left(A_{n} \neq 0\right)
$$

which are mutually orthogonal with respect to this inner product, that is,

$$
\left(p_{i}, p_{j}\right)=0 \quad, \quad \text { for } i \neq j
$$

These orthogonality conditions define the polynomials up to a multiplicative constant which for our purpose is set equal to unity.

The Gaussian quadrature roots $c_{K}(n)$ are the roots of $p_{n}(c)$. The Gaussian quadrature weights $H_{K}(n)$ are given by. 


$$
H_{K}{ }^{(n)}=\frac{\left(p_{n-1}, p_{n-1}\right)}{p_{n}{ }^{\prime}\left(c_{K}{ }^{(n)}\right) p_{n-1}\left(c_{K}{ }^{(n)}\right)}
$$

We are interested in evaluating an integral of form

$$
\int_{0}^{\infty} d c\left(c e^{-c^{2}}\right) h_{1}(c)
$$

where $h_{1}(c)$ is a polynomial in $c$ of degree 3 or less. Translated in the symbols involved in Eq. (D.1), $w_{1}(c)=c e^{-c^{2}}$ and $n=2$. Thus using Eq. (D.4), we get by successive calculations,

$$
\begin{aligned}
& p_{0}=1 \\
& p_{1}=c-\frac{\sqrt{\pi}}{2} \\
& p_{2}=\left\{c-\frac{\sqrt{\pi}}{2}\left(\frac{\pi-2}{4-\pi}\right)\right\} p_{1}-\left(1-\frac{\pi}{4}\right)
\end{aligned}
$$

Thus, the required Gaussian quadrature roots are the roots of the equation,

$$
p_{2}=0
$$

Solving, we get

$$
\begin{aligned}
c_{2}{ }^{(2)}, c_{1}{ }^{(2)} & =\frac{\pi^{1 / 2}}{2(4-\pi)} \pm \frac{\left(6 \pi^{2}-39 \pi+64\right)^{1 / 2}}{2(4-\pi)} \\
& \simeq 1.5181767,0.54664006
\end{aligned}
$$

Further, using (D.5), we get the following values for the Gaussian quadrature weights, 


$$
\begin{aligned}
H_{1}{ }^{(2)} & =\frac{\frac{1}{4}(4-\pi)^{3}}{\left(6 \pi^{2}-39 \pi+64\right)^{7 / 2}\left(\left(6 \pi^{2}-39 \pi+64\right)^{1 / 2}-\pi^{1 / 2}(\pi-3)\right)} \\
& =0.32523208
\end{aligned}
$$

and

$$
\begin{aligned}
H_{2}{ }^{(2)} & =\frac{\frac{1}{4}(4-\pi)^{3}}{\left(6 \pi^{2}-39 \pi+64\right)^{1 / 2}\left(\left(6 \pi^{2}-39 \pi+64\right)^{1 / 2}+\pi^{1 / 2}(\pi-3)\right)} \\
& =0.17476792
\end{aligned}
$$

In terms of the notation used in the text, $w_{a}=H_{7}{ }^{(2)}$, $w_{b}=H_{2}{ }^{(2)}, \xi_{a}=c_{1}{ }^{(2)}$ and $\xi_{b}=c_{2}{ }^{(2)}$. 